\title{
Astroglial Calcium Signaling Encodes Sleep Need in Drosophila
}

Ian D. Blum ${ }^{1 \#}$, Mehmet F. Keleş ${ }^{1 \#}$, El-Sayed Baz², Emily Han ${ }^{3}$ Kristen Park ${ }^{1}$, Skylar

Luu $^{1}$, Habon Issa ${ }^{1}$, Matt Brown ${ }^{3}$, Margaret C.W. Ho ${ }^{1}$, Masashi Tabuchi ${ }^{1,4}$, Sha Liu $^{2 *}$, and Mark N. $\mathrm{Wu}^{1,3^{* \wedge}}$

${ }^{1}$ Department of Neurology, Johns Hopkins University, Baltimore, MD 21205, U.S.A.

${ }^{2}$ VIB Center for Brain and Disease Research and Department of Neurosciences, KU

Leuven, Leuven, 3000, Belgium

${ }^{3}$ Solomon H. Snyder Department of Neuroscience, Johns Hopkins University, Baltimore, MD 21205, U.S.A.

${ }^{4}$ Present address: Department of Neurosciences, Case Western Reserve University, Cleveland, $\mathrm{OH} 44106$, U.S.A.

\#These authors contributed equally to this work

*Correspondence: sha.liu@kuleuven.vib.be or marknwu@jhmi.edu

${ }^{\wedge}$ Lead contact 


\section{SUMMARY}

Sleep is under homeostatic control, whereby increasing wakefulness generates sleep need and triggers sleep drive. However, the molecular and cellular pathways by which sleep need is encoded are poorly understood. In addition, the mechanisms underlying both how and when sleep need is transformed to sleep drive are unknown. Here, using ex vivo and in vivo imaging, we show in Drosophila that astroglial $\mathrm{Ca}^{2+}$ signaling increases with sleep need. We demonstrate that this signaling is dependent on a specific L-type $\mathrm{Ca}^{2+}$ channel and is required for homeostatic sleep rebound. Thermogenetically increasing $\mathrm{Ca}^{2+}$ in astrocytes induces persistent sleep behavior, and we exploit this phenotype to conduct a genetic screen for genes required for the homeostatic regulation of sleep. From this large-scale screen, we identify TyrRII, a monoaminergic receptor required in astrocytes for sleep homeostasis. TyrRII levels rise following sleep deprivation in a $\mathrm{Ca}^{2+}$-dependent manner, promoting further increases in astrocytic $\mathrm{Ca}^{2+}$ and resulting in a positive-feedback loop. These data suggest that TyrRII acts as a gate to enable the transformation of sleep need to sleep drive at the appropriate time. Moreover, our findings suggest that astrocytes then transmit this sleep need to the R5 sleep drive circuit, by upregulation and release of the interleukin-1 analog Spätzle. These findings define astroglial $\mathrm{Ca}^{2+}$ signaling mechanisms encoding sleep need and reveal dynamic properties of the sleep homeostatic control system.

Keywords: sleep, astrocyte, Drosophila, calcium, homeostatic 


\section{INTRODUCTION}

The regulation of sleep by homeostatic forces is one of its defining features, and yet how sleep need is sensed and transduced into sleep drive remains poorly understood. The analysis of sleep homeostasis is guided by engineering control principles which posit that homeostatic systems comprise at least 3 components: a sensor that receives information about the state variable, an integrator that computes the difference between this state variable and a setpoint, and a downstream effector that responds to the integrator and directly manipulates the state variable[1]. Although the signal detected by "sleep sensors" remains debated, there is compelling evidence that a key stimulus for triggering sleep need is neuronal activity[2, 3]. For instance, studies in rodents and humans have demonstrated that tasks which activate specific regions in the brain will then locally promote an increase in the amplitude of electroencephalographic slow-wave activity, an established marker of sleep need[4-6].

More than a century ago, Cajal proposed that astrocytes, a subtype of glial cells, modulate neural connectivity across the sleep/wake cycle[7]. Since that time, emerging data have suggested that astrocytes play a key role in the regulation of sleep[8-11]. However, whether and how astrocytes sense and discharge sleep need is enigmatic. A number of special features of astrocytes make them well-suited for serving as "sensors" of sleep need. Astrocytes effectively tile the entire brain, and their processes form an intimate network around synapses and locally sample neural activity[12-15]. In addition, $\mathrm{Ca}^{2+}$ signaling plays an important role in astrocyte function, and modulation of intracellular $\mathrm{Ca}^{2+}$ levels is a broadly used mechanism for computing information[13, 1620]. Finally, astrocytes appear to directly release neurotransmitters and other effector molecules ("gliotransmission") $[15,21,22]$ and, at least in vitro, have been shown to secrete sleep-promoting substances[23]. Thus, astrocytes could potentially sense sleep need-generating signals, perform relevant computations, and release signaling molecules to act on a downstream integrator circuit.

The morphology and functions of astrocytes are largely conserved in many animal species, including in the fruit fly Drosophila melanogaster[24]. In addition, sleep in Drosophila shares all defining behavioral criteria of sleep with mammals[25, 26]. Here, we use the fly model to characterize the role of astrocytes in sleep homeostasis and to 
delineate the mechanisms by which astroglial $\mathrm{Ca}^{2+}$ signals encode and transmit sleep need. Using ex vivo and in vivo imaging, we show that astrocytic $\mathrm{Ca}^{2+}$ levels vary with sleep need and demonstrate a critical role for this $\mathrm{Ca}^{2+}$ signaling in sleep homeostasis. Importantly, using a forward genetic approach, we identify Tyramine Receptor II (TyrRII), a monoaminergic receptor that is transcriptionally upregulated in astrocytes following sleep deprivation and which functions in a positive feedback $\mathrm{Ca}^{2+}$ loop to regulate sleep homeostasis. Our data further suggest that this astroglial $\mathrm{Ca}^{2+}$ signaling culminates in the upregulation of the secreted molecule Spätzle (Spz), the fly analog of mammalian interleukin 1 (IL-1); Spz then signals to a previously defined sleep drive circuit, to specifically control homeostatic rebound sleep. Together, these data support a model wherein astrocytes act as "sensors" of sleep need and encode sleep pressure under conditions of substantial sleep loss. Moreover, the underlying transcriptional and positive feedback signaling processes provide a mechanistic framework for conceptualizing the delay between and transformation of sleep need to sleep drive. 


\section{RESULTS}

\section{$\mathrm{Ca}^{2+}$ signaling in astrocytes correlates with sleep need and is necessary for sleep homeostasis}

On balance, wakefulness is associated with greater neuronal activity[3, 6, 27-31]. Since astrocytes sense and respond to increases in neuronal activity through $\mathrm{Ca}^{2+}$ signaling mechanisms[10, 12, 15, 18, 22, 32, 33], we first asked whether $\mathrm{Ca}^{2+}$ levels in astrocytes vary according to sleep need. To do this, we expressed the genetically-encoded $\mathrm{Ca}^{2+}$ indicator CaMPARI2[34] in astrocytes and examined ex vivo CaMPARI signal in two different neuropil regions (superior medial protocerebrum, SMP, and antennal lobe, AL) at ZT0-3 (Zeitgeber time 0-3), ZT12-15 (mild increase in sleep need), and ZT0-3 following $12 \mathrm{hrs}$ sleep deprivation (SD, strong increase in sleep need). Astrocytes exhibit distinct pools of intracellular $\mathrm{Ca}^{2+}$ (e.g., soma vs processes) that have different temporal kinetics and may participate in different signaling pathways[17, 18, 20,33]. Thus, we analyzed CaMPARI signals in both of these locations. As shown in Figures 1A, 1B, and $1 \mathrm{D}, \mathrm{Ca}^{2+}$ levels in the processes of astrocytes were elevated at ZT12-15, compared to ZT0-3, and further increased at ZT0-3 following SD. In the cell bodies, astrocytic $\mathrm{Ca}^{2+}$ levels were not elevated at ZT12-15 but were markedly elevated at ZT0-3 following SD (Figures $1 \mathrm{~A}, 1 \mathrm{C}$, and $1 \mathrm{E}$ ). To address whether the increases in astrocyte $\mathrm{Ca}^{2+}$ concentrations simply reflected a stress response to mechanical SD, we also examined CaMPARI signal at ZT12-15 following $12 \mathrm{hrs}$ of SD during the daytime. CaMPARI signals were not elevated under these conditions, compared to control flies at ZT12-15 (Figures 1B-1E). These data suggest that astrocyte intracellular $\mathrm{Ca}^{2+}$, particularly in the cell bodies, increases in a non-linear manner in response to time awake.

We next examined whether changes in astrocytic $\mathrm{Ca}^{2+}$ following SD could be observed in living flies. We performed in vivo imaging of $\mathrm{Ca}^{2+}$ signals in astrocytic processes in the SMP region using myristoylated GCaMP (myr-GCaMP6s). The frequency of $\mathrm{Ca}^{2+}$ transients in the astrocytic processes was significantly increased following SD (Figures 1F-1H, S1A and S1B), whereas the event size and peak intensity of these $\mathrm{Ca}^{2+}$ transients were similar between these two conditions (Figures $\mathrm{S} 1 \mathrm{C}$ and $\mathrm{S} 1 \mathrm{D})$. Taken together, our ex vivo and in vivo data argue that astrocytic $\mathrm{Ca}^{2+}$ signaling increases with greater sleep need. 
To address whether astrocytic $\mathrm{Ca}^{2+}$ signaling is required for the homeostatic regulation of sleep, we sought to identify a molecule that fluxes $\mathrm{Ca}^{2+}$ and is important for this process. We conducted a candidate RNAi miniscreen of $\mathrm{Ca}^{2+}$-related channels, transporters, and exchangers and assayed for changes in sleep homeostasis. Knockdown of an L-type $\mathrm{Ca}^{2+}$ channel subunit (Ca- $\left.\alpha 1 \mathrm{D}\right)$ selectively in astrocytes led to a pronounced reduction in sleep rebound following SD (Figure 2A). These findings were next confirmed after backcrossing and with an additional RNAi line targeting Ca- $\alpha 1 \mathrm{D}$. Knockdown of Ca- $\alpha 1 \mathrm{D}$ in astrocytes substantially reduced sleep recovery following SD (Figures 2B, 2C, and S2A) and led to a mild increase in baseline daily sleep time (Figure 2D) which was driven solely by an increase in nighttime sleep with no significant effects on sleep consolidation (Figures S2B-S2E). To address the functional role of Ca- $\alpha 1 \mathrm{D}$ in regulating astrocytic $\mathrm{Ca}^{2+}$ levels, we measured CaMPARI signal in astrocytes in the $\mathrm{AL}$ following SD. As shown in Figures 2E-2G, knockdown of Ca- $\alpha 1 \mathrm{D}$ suppressed the increased $\mathrm{Ca}^{2+}$ observed in astrocyte processes and cell bodies following SD. These data suggest that increases in astrocytic $\mathrm{Ca}^{2+}$ are required for normal homeostatic sleep rebound.

\section{Ectopic astroglial $\mathrm{Ca}^{2+}$ signaling triggers both acute and delayed increases in sleep behavior}

To examine the consequences of increasing $\mathrm{Ca}^{2+}$ levels within astrocytes on sleep behavior, we expressed the temperature-sensitive cation channel dTrpA1 in astrocytes. Interestingly, we found that elevating $\mathrm{Ca}^{2+}$ in astrocytes (alrm-GAL4) during the night led to two phenotypes: a rapid increase in nighttime sleep during the heat pulse and a persistent increase in daytime sleep following the cessation of the heat pulse. These two phenotypes were also observed using an additional astrocyte driver (R86E01-GAL4) and were reminiscent of the distinct phenotypes observed with activation of the ExF12 sleep effector circuit (R72G06-GAL4) and the R5 (previously termed R2) sleep drive circuit $(R 58 H 05-A D ; R 46 C 03-D B D)$, respectively (Figures 3A-3C)[35, 36]. We manually scored fly behavior using video to demonstrate that flies were truly inactive (and not grooming or feeding) during and after thermogenetic activation. These data 
demonstrated that immobility was specifically increased by these manipulations (Figure S3A). To further confirm that the immobility measured reflects sleep and not simply immobility or paralysis, we assessed arousal threshold. Both during and after thermogenetic activation of astrocytes, flies demonstrated an increased arousal threshold to mild and moderate stimuli but were fully responsive to strong stimuli (Figure S3B). Finally, to ensure that the sleep induced by thermogenetic activation of astrocytes is reversible, we quantified sleep for several days after heat treatment (Figures S3C-S3E). In general, sleep normalizes by the night directly following the "sleep rebound," with the exception of a decrease of daytime sleep the following day ("Day 2"), which may represent "negative sleep rebound", a phenomena previously observed in both mammals and flies[37-39].

We next asked whether this persistent "sleep rebound" could be induced by a shorter period of astrocyte activation, similar to our previous findings with the R5 sleep drive circuit[36]. Indeed, $1 \mathrm{hr}$ heat activation of dTrpA1 in astrocytes also triggered a persistent increase in sleep, suggestive of an increase in sleep drive. Interestingly, however, unlike the case for R5 neurons where a monophasic increase in sleep was induced following the heat treatment[36], $1 \mathrm{hr}$ activation of astrocytes led to a biphasic sleep response - a rapid increase in sleep during the heat treatment followed by a delayed increase in sleep after the heat treatment (Figures 3D-3F). Similar observations were made using R86E01-GAL4 (Figures $\mathrm{S} 3 \mathrm{~F}$ and $\mathrm{S} 3 \mathrm{G}$ ). These data suggest that activation of astrocytes is sufficient for inducing sleep behavior and generating homeostatic sleep drive.

Astrocytes release various neurotransmitters and signaling molecules to modulate synaptic function[14, 15, 21, 22]. What effect might $\mathrm{Ca}^{2+}$ signaling in astrocytes have on neural activity? To address this, we performed patch-clamp recordings of the large ventrolateral clock neurons (1-LNvs). As clock neurons that modulate arousal[40-42], the firing rate of the 1-LNvs is under circadian control[43-45], but their sensitivity to sleep need in unknown. Therefore, we first examined whether l-LNv firing is altered following $12 \mathrm{hrs}$ of SD; indeed, their firing rate at ZT0-2 was significantly reduced and their resting membrane potential was hyperpolarized after SD (Figures $3 \mathrm{G}, 3 \mathrm{H}$, and $\mathrm{S} 4 \mathrm{~A}$ ). We then assessed the impact of dTrpA1 activation of astrocytes on 1-LNv activity. alrm $>d \operatorname{TrpA1-}$ 
mediated astrocyte activation led to a significant reduction in spiking frequency and resting membrane potential, whereas heat treatment alone (UAS-dTrpAl) had no effect on firing rate (Figures 3I-3K, S4B, and S4C). Similar observations were made using R86E01-GAL4 (Figures S4D and S4E). These data suggest that activation of astrocytes can result in inhibition of arousal-promoting systems.

\section{TyrRII is required for astroglial control of sleep homeostasis}

Little is known about the molecular pathways by which astrocytes regulate sleep. The use of forward genetic screens to identify novel genes critical for sleep homeostasis has been hindered by the difficulty of performing sleep deprivation robustly and reproducibly on a large-scale. To circumvent this problem and identify astrocytic genes required for sleep homeostasis, we capitalized on our finding of persistent sleep drive following astrocyte activation and performed a screen for genes required for this phenotype. From a screen of $\sim 3,200$ RNAi lines, we identified $\sim 40$ lines that reproducibly suppressed this sleep phenotype (Figure 4A). Interestingly, we found that knockdown of the largely uncharacterized receptor TyrRII markedly suppressed the persistent sleep phenotype seen with activation of astrocytes (Figures 4A-4C). To address whether TyrRII expression in astrocytes is directly required for the homeostatic regulation of sleep, we assessed sleep recovery following $12 \mathrm{hrs}$ of mechanical SD. Sleep recovery, but not baseline daily sleep, was significantly reduced when TyrRII was knocked down in astrocytes, compared to controls (Figures 4D-4F and S5A). In contrast, knockdown of TyrRII in astrocytes did not yield consistent effects on baseline daytime sleep, nighttime sleep, or sleep consolidation (Figures S5B-S5E).

\section{TyrRII is upregulated with sleep loss and participates in a positive feedback $\mathrm{Ca}^{2+}$ - signaling mechanism}

TyrRII has previously been shown to be broadly responsive to a variety of monoamines and, given that monoamine release is generally associated with arousal[46-49], this system could represent an elegant mechanism for astrocytes to track wakefulness and consequently homeostatic sleep need. Because monoaminergic receptor expression is often tightly regulated by diverse signaling mechanisms[50], we examined whether tyrRII 
expression varied according to sleep need. We assessed astrocyte expression of tyrRII mRNA using TRAP-qPCR (Translating Ribosome Affinity Purification followed by Quantitative PCR)[51], which we performed by expressing eGFP-RpL10a in astrocytes and immunopurifying actively translating mRNA using magnetic beads coated with antiGFP antibodies (Figure 5A). As expected, immunoprecipitates were dramatically enriched for a glial marker (repo), compared to a neuronal marker ( $n S y b)$ (Figure 5B). Interestingly, tyrRII mRNA was markedly elevated $\sim 10$-fold after sleep deprivation (Figure 5C). This increase could be recapitulated by dTrpAl activation of astrocytes, suggesting that the sleep need-dependent increase in tyrRII is $\mathrm{Ca}^{2+}$-dependent (Figures $5 \mathrm{D}$ and $5 \mathrm{E})$.

We next asked whether TyrRII protein levels were also increased following sleep deprivation. To address this question, we used a MiMIC transposon insertion[52], where GFP is fused in-frame into the $2^{\text {nd }}$ extracellular loop of the TyrRII protein. First, we assayed baseline and rebound sleep in homozygote Mi\{PT-GFSTF.2\}TyrRII ${ }^{M I 12699}$ flies and found no differences compared to heterozygous controls, suggesting that the GFP insertion does not disrupt protein function or localization (Figures S5F-S5H). Consistent with our findings with tyrRII transcript, TyrRII::GFP expression was substantially increased following $12 \mathrm{hr}$ SD vs non-SD controls, both in terms of number and size of puncta (Figures S5I-S5K). Interestingly, super-resolution microscopy revealed that the sleep deprivation-mediated increase in TyrRII-GFP expression within the AL neuropil was largely localized to bulbous structures within astrocytic processes (Figure S5L). These structures were observed by R86E01-GAL4-driven tdTomato expression independent of sleep need-state, suggesting that they are not a direct result of either sleep deprivation or the expression of TyrRII::GFP.

Our previous data suggested that the upregulation of tyrRII transcript following $\mathrm{SD}$ is dependent on astrocytic intracellular $\mathrm{Ca}^{2+}$ (Figure 5E). Moreover, thermogenetic activation of astrocytes resulted in a marked increase in TyrRII::GFP puncta number and a significant, but less pronounced, increase in puncta size (Figures 6A-6C). To directly test whether astrocytic $\mathrm{Ca}^{2+}$ signaling is required for the sleep need-induced elevation in TyrRII::GFP, we performed RNAi knockdown of Ca- $\alpha 1 \mathrm{D}$ in astrocytes and quantified TyrRII::GFP expression before and after SD. As shown in Figures 6D-6F, TyrRII::GFP 
expression following SD, but not under baseline conditions, was significantly reduced with concomitant knockdown of Ca- $\alpha 1 \mathrm{D}$, compared to controls. Prior studies have shown that monoaminergic signaling induces $\mathrm{Ca}^{2+}$ elevations in astrocytes in both flies and mice[53,54]. Thus, TyrRII might produce an amplifying positive-feedback loop in astrocytes-- not only does TyrRII expression depend on intracellular $\mathrm{Ca}^{2+}$ levels, but the higher levels of TyrRII expression following SD contribute to further increases in $\mathrm{Ca}^{2+}$ levels. To address this possibility, we examined whether loss of TyrRII suppressed the elevation of intracellular $\mathrm{Ca}^{2+}$ seen in astrocytes following SD. Following SD, CaMPARI signal in both astrocyte processes and cell bodies was substantially reduced in R86E01-GAL4>UAS-TyrRII-RNAi animals, compared to controls (Figures 6G-6I).

Taken together, these data suggest that, as sleep need accrues during protracted arousal, TyrRII amplifies $\mathrm{Ca}^{2+}$ signaling in a positive-feedback manner, priming astrocytes and sensitizing them to monoamines.

\section{Astrocyte-derived Spätzle transmits sleep need to the R5 sleep drive circuit}

Not only are the underlying molecular pathways unclear, but the circuit mechanisms by which astrocytes signal sleep need are also unknown. In principle, such signaling could occur locally to neurons throughout the brain. Alternatively, sleep need could be integrated and transmitted to a central neural circuit. We first asked whether a specific signaling molecule is released from astrocytes to convey sleep need. spätzle (spz), the Drosophila analog of IL-1, was a "hit" in our dTrpA1-mediated RNAi screen, and, because IL-1 has previously been implicated in the homeostatic regulation of sleep in mammals[55], we focused on this gene. We found that knockdown of spz in astrocytes significantly reduced sleep recovery following SD (Figures 7A, 7B, and S6A), while not affecting daily sleep time, daytime sleep, nighttime sleep, or sleep consolidation under baseline conditions (Figure 7C and S6B-S6E). We next investigated whether spz expression was altered in response to changes in sleep need. We again performed TRAPqPCR and found that $s p z$ transcript was increased $\sim 9$-fold in astrocytes following SD (Figure 7D) or thermogenetic activation (Figure S6F), supportive of a role for astrocytic $\mathrm{Spz}$ in relaying sleep need. 
We previously identified a neural circuit, comprising R5 ellipsoid body (EB) ring neurons, that encodes sleep drive[36]; we hypothesized that astrocytes may convey information regarding sleep need to these neurons. Moreover, recent data suggest that Toll, the receptor for Spz, is enriched within the EB in the adult brain[56]. Knockdown of Toll in R5 neurons led to a significant reduction in sleep recovery following SD (Figures 7E, 7F, and S6G), but not daily sleep time, or daytime sleep, nighttime sleep, and sleep consolidation under baseline conditions (Figures 7G and S6H-S6K). We previously demonstrated that intracellular $\mathrm{Ca}^{2+}$ levels in $\mathrm{R} 5$ are elevated with greater sleep need and that this higher intracellular $\mathrm{Ca}^{2+}$ was critical for the synaptic plastic changes encoding sleep drive in these neurons[36]. Thus, we assessed whether thermogenetic activation of astrocytes would lead to a similar increase in R5 intracellular $\mathrm{Ca}^{2+}$. To do this, we first generated a QF2 driver line broadly labeling astrocytes (alrmQF2) (Figures S7A and S7B). Importantly, we found that dTrpA1 activation of astrocytes led to a substantial elevation of GCaMP signal in the R5 neurons (Figures 7H and 7I). These findings suggest that astrocytes sense substantial sleep need and convey this information, by upregulating and releasing Spz. In addition, our data support the hypothesis that Spz then activates Toll receptor in R5 neurons to generate sleep drive.

\section{DISCUSSION}

Although astrocytes have been implicated in the homeostatic regulation of sleep[8], their specific role and the underlying mechanisms have been unresolved. Our data support a role for astrocytes as sensors of sleep need and define signaling mechanisms within these cells that mediate the integration and transmission of this information to a downstream homeostatic sleep circuit (Figure 7J). In this model, neural activity is sensed by astrocytic processes, leading to an increase in $\mathrm{Ca}^{2+}$ levels, which depends at least in part on specific L-type Voltage-Gated $\mathrm{Ca}^{2+}$ channels (VGCC)[57-60]. Interestingly, while astrocytes have been shown to exhibit hyperpolarized membrane potentials with small depolarizations[57], this particular subtype of L-type VGCC can be activated at substantially lower membrane potentials than other members of this channel family[61].

As the increased neural activity persists, $\mathrm{Ca}^{2+}$-mediated transcription of TyrRII is induced in astrocytes. TyrRII is relatively unstudied, but in vitro data suggest that it 
responds non-specifically to multiple monoamines[62]. Thus, its upregulation in astrocytes should sensitize these cells to signaling via monoamines, which are intimately associated with wakefulness[63]. The requirement for monoamines in this pathway may provide a logic gate for the system, imparting specificity to the signaling mechanism acting downstream of neural activity, whose semantic properties may be too broad. TyrRII itself is required for further $\mathrm{Ca}^{2+}$ elevations, leading to a positive-feedback loop.

Our data suggest that this amplification of astrocytic $\mathrm{Ca}^{2+}$ signals results in transcriptional upregulation of $s p z$, the fly analog of IL-1. There is an accumulating body of evidence implicating IL-1 in sleep homeostasis in mammals[55, 64-66], and our findings demonstrating a functional role for astrocytic Spz in sleep homeostasis demonstrate that these mechanisms are conserved from invertebrates to vertebrates. Our data suggest that, under conditions of strong sleep need, Spz is released from astrocytes and transmits this information by signaling to a central sleep drive circuit (the R5 neurons) to promote homeostatic sleep "rebound."

From a broader perspective, our model draws attention to a fundamental, yet poorly understood, aspect of sleep homeostasis — how a highly dynamic input (i.e., neural activity operating on millisecond timescale) is integrated and transformed to generate a sleep homeostatic force that functions on a significantly slower timescale. Although the precise identity of the signals embodying sleep need remain unclear, there is substantial experimental and conceptual support for the notion that neural activity increases with wakefulness[67] and is a key trigger for this process[3, 28]. Yet, the dynamic mechanisms by which this neural activity, and, by extension, sleep need is transformed to sleep drive are unknown. The homeostatic regulation of processes and behaviors involving bistable states, such as sleep vs wakefulness, requires a prominent delay between the detection of the disturbance and the generation of the response[68, 69]. In addition, the stability and switching between such bistable states can be facilitated by positive feedback loops[46, 70-72]. We propose that the transcription/translation of TyrRII, coupled with the generation of a positive-feedback loop, provide a timing delay followed by a more rapid elevation in astroglial $\mathrm{Ca}^{2+}$ after reaching a set threshold, thus enabling a non-linear response to the continual sampling of sleep need. The 
transcriptional/translation upregulation of Spz could represent an additional layer of delay.

While the sleep homeostat has been implicated in regulating sleep under both baseline conditions and following substantial sleep loss[73], the astrocytic TyrRII/Spz $\rightarrow$ R5 Toll pathway selectively regulates sleep "rebound" only after protracted sleep deprivation, as previously shown for the R5 sleep drive circuit itself[36]. Thus, this pathway likely represents a distinct process for conveying potent sleep need to generate robust sleep drive. Our findings highlight the importance and enigmatic nature of these processes for the homeostatic control of baseline sleep and suggest that similar dynamic control mechanisms may also be relevant under baseline conditions.

\section{ACKNOWLEDGEMENTS}

We thank M. Freeman, R. Jackson, T. Littleton, C. Potter, G. Rubin, and E. Schreiter for kindly sharing reagents. We also thank the Bloomington Stock Center (supported by NIH grant P40OD018537), the Vienna Drosophila Stock Center (www.vdrc.at), and the TRiP at Harvard Medical School (supported by NIH grant R01GM084947) for fly stocks used in this study. We thank members of the Wu Lab for discussion. This work was supported by NINDS Center Grant P30 NS050274 for use of the Core Machine Shop and the Multiphoton Imaging Core, ERC Starting Grant 758580 (S.L.), and NIH grants K99NS101065 (M.T.), R01NS094571-03S1 (M.N.W.), R01NS100792 (M.N.W.), and R01NS079584 (M.N.W.).

\section{AUTHOR CONTRIBUTIONS}

Conceptualization, IDB, SL, MNW; Methodology, IDB, MFK, EH, MT, MNW;

Software, IDB, MFK; Investigation, IDB, MFK, E-SB, EH, KP, SL, HI, MCW, MB, MT;

Writing - Original Draft, IDB, MNW; Writing - Review \& Editing, IDB, MFK, E-SB, EH, KP, SL, HI, MB, MCW, MT, SL, MNW; Resources, SL, MNW; Visualization, IDB, MFK, EH, E-SB; Supervision, IDB, MT, SL, MNW; Funding Acquisition, MT, SL, MNW. 
bioRxiv preprint doi: https://doi.org/10.1101/2020.07.04.187906; this version posted July 5, 2020. The copyright holder for this preprint (which was not certified by peer review) is the author/funder. All rights reserved. No reuse allowed without permission.

\section{DECLARATION OF INTERESTS}

The authors declare no competing interests. 


\section{FIGURE LEGENDS}

\section{Figure 1. $\mathrm{Ca}^{2+}$ signaling in astrocytes correlates with sleep need.}

(A) Representative confocal images of pre-photoconversion (Pre-PC) and postphotoconversion (Post-PC) CaMPARI2 signal in the antennal lobe (AL) at ZT0 in the presence or absence of sleep deprivation (SD) from R86E01-GAL4>UAS-CaMPARI2L398T flies.

(B-E) CaMPARI2 signal (Fold R/G) from astrocyte processes (B and D) or cell bodies (C and E) from Superior Medial Protocerebrum (SMP) or AL from R86E01-GAL4>UASCaMPARI2-L398T flies at ZT0-3 or ZT12-15 under baseline conditions (AL: $\mathrm{n}=5$ for ZT0-3 and $n=6$ for ZT12-15; SMP: $n=5$ for ZT0-3 and $n=6$ for ZT12-15) or after 12 hrs of SD (AL: $\mathrm{n}=6$ for ZT0-3 and ZT12-15; SMP: $\mathrm{n}=6$ for ZT0-3 and ZT12-15).

(F and G) Representative 2-photon images (left) and event traces (right) of SMP from control (F) and sleep-deprived (G) R86E01-GAL4>UAS-myr-GCaMP6s flies at ZT0-2. Each image is the mean intensity of an entire recording from in vivo 2-photon $\mathrm{Ca}^{2+}$ imaging experiments. Data-driven regions of interest (ROIs) that were used to extract event traces are highlighted in white. Corresponding traces and ROIs are numbered. (H) Frequency of events for control (gray) and sleep-deprived (magenta) flies ( $n=9$ flies for control and $n=7$ flies for SD) expressing membrane-bound GCaMP6s in astrocytes. Scale bars denote $10 \mu \mathrm{m}$ in all images. For all bar graphs throughout manuscript, mean \pm SEM is shown. In this and subsequent Figures, “*”, “**”, “***”, and "ns" denote $P<0.05$, $P<0.01, P<0.001$, and not significant, respectively.

\section{Figure 2. $\mathrm{Ca}^{2+}$ signaling in astrocytes is required for sleep homeostasis.}

(A) Mechanical deprivation miniscreen of astrocyte $\mathrm{Ca}^{2+}$-related channels, transporters, and exchangers. Relative change in sleep rebound for R86E01-GAL4>UAS-RNAi flies expressed as a percentage of sleep rebound observed in R86E01-GAL4>UAS-empty vector flies.

(B) Sleep recovery curves for R86E01-GAL4>ctrl (gray) vs. R86E01-GAL4>UAS-Ca$\alpha 1 D$-RNAi\#1 (green) and R86E01-GAL4>UAS-Ca- $\alpha 1 D-R N A i \# 2$ (magenta) flies. 
(C and D) Sleep recovered (\%) (C) and daily sleep amount (D) for R86E01-GAL4>ctrl (n=103), R86E01-GAL4>UAS-Ca- $\alpha 1 D-R N A i \# 1(\mathrm{n}=53)$, and R86E01-GAL4>UAS-Ca$\alpha 1 D-R N A i \# 2(\mathrm{n}=35)$ flies.

(E) Pixel-by-pixel heatmap of CaMPARI2 photoconversion signal in the AL region at ZT0-3 following $12 \mathrm{hr} \mathrm{SD}$ in R86E01-GAL4>UAS-CaMPARI2-L398T flies, in the presence or absence of $U A S-C a-\alpha 1 D-R N A i \# 1$. Dotted squares highlight $\mathrm{Ca}^{2+}$ signals from fine astrocyte processes, and white arrows denote cell bodies. Scale bars denote 10 $\mu \mathrm{m}$.

(F and G) Quantification of average CaMPARI2 signal (Fold R/G) from ROIs targeting astrocyte processes $(F)$ or cell bodies $(\mathrm{G})$ represented in $(\mathrm{E})$.

\section{Figure 3. Astrocyte activation induces both proximate and delayed sleep and inhibits neural activity.}

(A) Sleep profile of $c t r l>U A S-d T r p A 1$ flies (gray) vs $R 58 H 05-A D ; R 46 C 03-D B D>U A S$ $d \operatorname{Trp} A 1$ (blue, upper panel), R72G06-GAL4>UAS-dTrpAl (green, middle panel) or alrmGAL4>UAS-dTrpAl flies (magenta, lower panel). Highlighted period denotes $12 \mathrm{hr}$ dTrpA 1 activation at $28^{\circ} \mathrm{C}$. Note that the controls in the 3 sleep profiles are from the same dataset.

(B and C) Sleep amount over a $12 \mathrm{hr}$ period during (B) or $6 \mathrm{hr}$ after (C) dTrpA1 activation for $c t r l>U A S-d \operatorname{Trp} A 1(\mathrm{n}=81), R 58 H 05-A D ; R 46 C 03-D B D>U A S-d T r p A 1$ $(\mathrm{n}=17), R 72 G 06-G A L 4>U A S-d T r p A 1(\mathrm{n}=51), R 86 E 01-G A L 4>U A S-d T r p A 1(\mathrm{n}=29)$, and alrm-GAL4>UAS-dTrpA1 ( $\mathrm{n}=40)$.

(D) Sleep profile of $c t r l>U A S$-dTrpA1 (gray) and alrm-GAL4>UAS-dTrpA1 (magenta). Highlighted period denotes $1 \mathrm{hr} \operatorname{dTrpA} 1$ activation at $31^{\circ} \mathrm{C}$.

(E and F) Sleep amount during (E) and after (F) $1 \mathrm{hr}$ dTrpA1 activation for $c t r l>U A S$ $\operatorname{dTrpA1}(\mathrm{n}=32)$, alrm-GAL4>ctrl $(\mathrm{n}=32)$, and alrm-GAL4>UAS-dTrpA1 $(\mathrm{n}=28)$ flies. $(\mathrm{G}$ and $\mathrm{H})$ Representative whole-cell recording traces $(\mathrm{G})$ and mean firing rate $(\mathrm{H})$ of spontaneous action potentials from 1-LNvs in $P D F-G A L 4>U A S-C D 8:: G F P$ flies from ZT0-2 under baseline ("ctrl”, $\mathrm{n}=8$ ) or $12 \mathrm{hr}$ sleep-deprived ("SD”, n=8) conditions. (I-K) Representative whole-cell recording traces (I) and mean firing rate of spontaneous action potentials of 1-LNvs in alrm-GAL4>UAS-dTrpA1;PDF-LexA>LexAop2- 
CD2::GFP (n=9) (J) or alrm-GAL4>ctrl, PDF-LexA>LexAop2-CD2::GFP $(\mathrm{n}=8)(\mathrm{K})$ flies from ZT0-2 at $22^{\circ} \mathrm{C}$ ("RT") and $29^{\circ} \mathrm{C}$ ("+heat").

Figure 4. TyrRII is required in astrocytes for homeostatic sleep rebound.

(A) Histogram for RNAi genetic screen for alrm-GAL4>UAS-dTrpA1, UAS-RNAi flies

( $n=3,150$ genes, $n=4$ flies per genotype) showing "rebound" sleep (6 hr post-activation) induced by $1 \mathrm{hr}$ of heat $\left(31^{\circ} \mathrm{C}\right)$ from ZT0-1. The amount of "rebound sleep" for alrmGAL4>UAS-TyrRII-RNAi\#1-expressing flies is noted. Bars in gray denote values lying $+/-2.5 \mathrm{SD}$ from the mean.

(B) Sleep profile of alrm-GAL4>UAS-dTrpA1, ctrl (gray), and alrm-GAL4>UASdTrpA1, UAS-TyrRII-RNAi\#l (green) flies. Highlighted period denotes $12 \mathrm{hr}$ dTrpA1 activation at $28^{\circ} \mathrm{C}$.

(C) Sleep amount for the $6 \mathrm{hr}$ period after dTrpA1 activation for alrm-GAL4>UASdTrpA1, ctrl (n=19), and alrm-GAL4>UAS-dTrpA1, UAS-TyrRII-RNAi\#1 (n=24).

(D) Sleep recovery curves for R86E01-GAL4>ctrl (gray) vs R86E01-GAL4>UASTyrRII-RNAi\#1 (green) and R86E01-GAL4>UAS-TyrRII-RNAi\#2 (magenta) flies after overnight (12 hr) SD.

(E and F) Sleep recovered (\%) (E) and daily sleep amount (F) for R86E01-GAL4>ctrl $(\mathrm{n}=81)$ and R86E01-GAL4>UAS-TyrRII-RNAi \#1 $(\mathrm{n}=33)$, and R86E01-GAL4>UASTyrRII-RNAi \#2 ( $\mathrm{n}=36)$.

Figure 5. tyrRII mRNA is upregulated with sleep deprivation and astrocyte activation

(A) Schematic of translating ribosomal affinity purification (TRAP) procedure for isolating actively translating mRNA from genetically-defined astrocytes in whole fly heads.

(B) Relative ratio of astrocyte marker (repo) and neural marker ( $n S y b)$ mRNA level in whole head flowthrough (input, $n=3$ replicates) vs astrocyte-TRAP (pulldown, $n=3$ replicates) samples from sleep deprivation experiment. 
(C) Relative change in tyrRII mRNA level in sleep-deprived (“SD”, n=3 replicates) vs non-sleep deprived (“no SD”, n=3 replicates) flies from astrocyte-TRAP (pulldown) vs whole head (input) samples.

(D) Relative ratio of astrocyte marker (repo) and neural marker ( $n S y b)$ mRNA level in whole head flowthrough (input, $n=3$ replicates) vs astrocyte-TRAP (pulldown, $n=3$ replicates) samples from experiments where astrocytes are thermogenetically activated.

(E) Relative fold change in tyrRII mRNA level in thermogenetically activated alrmGAL4 $>U A S$-dTrpAl (n=3 replicates) vs ctrl $>U A S$-dTrpA1 ("ctrl", n=3 replicates) flies from astrocyte-TRAP (pulldown) vs whole head (input) samples.

\section{Figure 6. Astrocytic TyrRII is upregulated with sleep need and participates in a positive-feedback calcium signaling mechanism}

(A) Representative images of TyrRII::GFP signal at the AL from alrm $>d \operatorname{TrpA1}, M i\{P T$ GFSTF.2\}TyrRII ${ }^{M I 12699 /+}$ or ctrl>UAS-dTrpA1, Mi\{PT-GFSTF.2\}TyrRII ${ }^{M I 12699 /+}$ flies.

Whole-mount brains were immunostained with anti-GFP (green) and anti-BRP (magenta) at ZT0-1 in the presence $($ alrm $>d \operatorname{TrpA1})$ or absence $(\operatorname{ctrl}>d \operatorname{Trp} A 1)$ of thermogenetic activation ("+Heat", $12 \mathrm{hrs}$ at $28^{\circ} \mathrm{C}$ ).

(B and C) Number (B) and size (C) of TyrRII::GFP puncta in the AL at ZT1 in the presence (alrm $>d \operatorname{TrpA1}$ ) or absence (ctrl>dTrpAl) of before ("Pre") and after ("Post") thermogenetic activation ( $\mathrm{n}=5$ for all groups and conditions).

(D) Representative images of TyrRII::GFP signal at the AL following $12 \mathrm{hr}$ sleep deprivation from ctrl>UAS-Ca- $\alpha 1 D$ RNAi\#1, Mi\{PT-GFSTF.2\}TyrRII ${ }^{M I 12699} /+$ or R86E01-GAL4>UAS-Ca- $\alpha 1 D$ RNAi\#1, Mi\{PT-GFSTF.2\}TyrRII MI12699/+ flies. Wholemount brains were collected from ZT0-1 and immunostained with anti-GFP (green) and anti-BRP (magenta).

(E and F) Number (E) and size (F) of TyrRII::GFP puncta in the AL under baseline (ZT0) or SD conditions with (magenta) or without (gray) $C a-\alpha 1 D$ knockdown ( $\mathrm{n}=8$ for all groups and conditions).

(G) Pixel-by-pixel heatmap of CaMPARI2 photoconversion signal in the AL region from ZT0-3 following $12 \mathrm{hr}$ SD in R86E01-GAL4>UAS-CaMPARI2-L398T flies, in the presence $(\mathrm{n}=9)$ and absence $(\mathrm{n}=10)$ of $U A S-T y r R I I-R N A i \# 2$. 
(H and I) Quantification of CaMPARI2 signal (Fold R/G) from astrocyte processes $(\mathrm{H})$ or cell bodies (I). Scale bars denote $20 \mu \mathrm{m}$ in (A) and (D) and $10 \mu \mathrm{m}$ in (G).

Figure 7. Astrocyte-derived Spätzle, an IL-1 analog, signals sleep need to the R5 sleep drive circuit via the Toll receptor.

(A) Sleep recovery curve for $R 86 E 01-G A L 4>c t r l$ (gray), R86E01-GAL4>UAS-spz-

RNAi\#1 (green), and R86E01-GAL4>UAS-spz-RNAi\#2 (magenta) flies.

(B and C) Sleep recovered (\%) (B) and daily sleep amount (C) for R86E01-GAL4>ctrl $(\mathrm{n}=46)$, R86E01-GAL4>UAS-spz-RNAi\#1 (n=40), and R86E01-GAL4>UAS-spz-RNAi\#2 $(\mathrm{n}=56)$ flies.

(D) Relative change in $s p z$ mRNA level in sleep-deprived (“SD”, $\mathrm{n}=3$ replicates) vs nonsleep deprived ("no SD”, n=3 replicates) flies from astrocyte-TRAP (pulldown) vs whole head (input) samples.

(E) Sleep recovery curve of $R 58 H 058-G A L 4>c t r l$ (gray), R58H05-GAL4>UAS-Toll$R N A i$ (green), and R58H05-GAL4>UAS-Toll-miR (magenta).

(F and G) Sleep recovered (\%) (F) and daily sleep amount (G) for R58H05-GAL4>ctrl $(\mathrm{n}=103), R 58 H 05-G A L 4>U A S-T o l l-R N A i(\mathrm{n}=51)$, and R58H05-GAL4>UAS-Toll-miR $(\mathrm{n}=56)$ flies.

(H and I) Representative images of GCaMP (upper panels) and tdTomato (lower panels) fluorescence intensity $(\mathrm{H})$ and relative GCaMP fluorescence intensity (I) in the R5 ring of ctrl $>Q U A S-d T r p A 1 ;$ R58H05-GAL4>UAS-GCaMP6s, UAS-CD4::tdTomato (ctrl, n=5) vs alrm-QF2>QUAS-dTrpA1; R58H05-GAL4>UAS-GCaMP6s, UAS-CD4::tdTomato $(\mathrm{n}=4)$ flies at ZT3-4 after $12 \mathrm{hrs}$ of heat treatment from ZT12-24 at $28^{\circ} \mathrm{C}$. For $(\mathrm{H})$, dashed lines indicate the R5 ring, and scale bar denotes $20 \mu \mathrm{m}$.

(J) Model for astroglial $\mathrm{Ca}^{2+}$ signaling in the homeostatic regulation of sleep. Neural activity during wakefulness is sensed by local astrocytes, resulting in increased $\mathrm{Ca}^{2+}$ in the processes, which requires specific voltage-gated $\mathrm{Ca}^{2+}$ channels ("Baseline"). Sleep loss generates protracted calcium signaling and leads to upregulation of TyrRII, sensitizing astrocytes to the actions of monoamines that are associated with wakefulness and further increasing $\mathrm{Ca}^{2+}$ levels in these cells ("Priming"). When sufficient sleep need has accumulated, as measured by heightened levels of astroglial $\mathrm{Ca}^{2+}$, 
bioRxiv preprint doi: https://doi.org/10.1101/2020.07.04.187906; this version posted July 5, 2020. The copyright holder for this preprint (which was not certified by peer review) is the author/funder. All rights reserved. No reuse allowed without permission.

transcription/translation of Spz is upregulated ("Potentiated"). Spz is then released and acts on Toll receptors in the R5 neurons to trigger global sleep drive. 


\section{SUPPLEMENTAL DATA}

Figure S1. $\mathrm{Ca}^{2+}$ signaling in astrocytes correlates with sleep debt, Related to Figure 1

(A and B) Additional representative examples of in vivo GCaMP 2-photon images (left) and event traces (right) of SMP from control (A) and sleep-deprived (B) R86E01GAL4>UAS-myr-GCaMP6s flies.

(C and D) Event size (C), and maximum $\Delta F / F(D)$ for control (gray, $n=56$ ) and sleepdeprived (magenta, $n=74$ ) flies expressing membrane-bound GCaMP6s in astrocytes.

Figure S2. $\mathrm{Ca}^{2+}$ signaling in astrocytes is required for sleep homeostasis, Related to Figure 2

(A) Average $24 \mathrm{hr}$ sleep profiles from baseline and post-sleep deprivation days overlaid

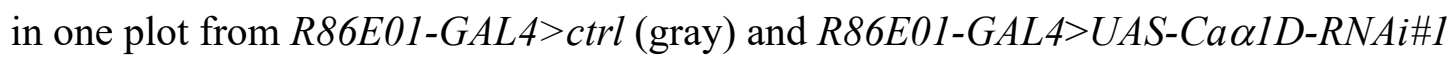
(green) flies. White and black bars indicate $12 \mathrm{hr}$ light and dark periods.

(B-E) Daytime sleep time (B) nighttime sleep time (C), nighttime sleep bout duration (D), and nighttime sleep bout number (E) under baseline conditions for R86E01GAL4>ctrl, R86E01-GAL4>UAS-Ca- $11 D-R N A i \# 1$, and R86E01-GAL4>UAS-Ca- $11 D-$ RNAi\#2 flies. Note that the data shown in panels (A-E) are from the same animals shown in Figures 2B-2D.

Figure S3. Additional behavioral characterizing sleep upon thermogenetic activation of astrocytes, Related to Figure 3

(A) Manual behavioral scoring of $1 \mathrm{hr}$ of high resolution videos of $c t r l>U A S-d T r p A 1$ (left, $\mathrm{n}=12$ ) and alrm-GAL4>UAS-dTrpAl (right, $\mathrm{n}=12$ ) flies during thermogenetic activation of astrocytes (ZT21-22, $28^{\circ} \mathrm{C}$, top) and after the temperature is returned to baseline conditions (ZT2-3, $22^{\circ} \mathrm{C}$, bottom).

(B) Percentage of ctrl $>U A S-d \operatorname{Trp} A 1(\mathrm{n}=28)$, alrm-GAL4>UAS-dTrpA1 $(\mathrm{n}=28)$, or alrmGAL4 (n=29) flies aroused by a mild, moderate, or strong mechanical stimulus during thermogenetic activation of astrocytes $\left(\mathrm{ZT} 15-21,28^{\circ} \mathrm{C}\right.$, left) and after the temperature is returned to baseline conditions (ZT1-7, $22^{\circ} \mathrm{C}$, right). 
(C) Sleep profile of alrm-GAL4>ctrl (gray), alrm-GAL4>UAS-dTrpAl (blue) or $c t r l>U A S-d \operatorname{Trp} A 1$ (purple) flies. Highlighted period denotes $12 \mathrm{hr}$ dTrpA1 activation at $28^{\circ} \mathrm{C}$.

(D and E) Total nighttime (D) or daytime (E) sleep amounts across experimental days for ctrl $>U A S-d \operatorname{TrpA1}(\mathrm{n}=32)$, alrm-GAL4>UAS-dTrpA1 $(\mathrm{n}=32)$, or alrm-GAL4>ctrl $(\mathrm{n}=31)$ flies shown in $(\mathrm{C})$.

(F and G) Sleep amount during (F) and after $(\mathrm{G}) 1 \mathrm{hr}$ dTrpA1 activation from R86E01GAL4>ctrl $(\mathrm{n}=16)$, and R86E01-GAL4>UAS-dTrpA1 (n=32) flies.

\section{Figure S4. Additional electrophysiological data, Related to Figure 3}

(A) Resting membrane potential of l-LNvs is significantly reduced after $12 \mathrm{hrs}$ of overnight sleep deprivation. The data shown in (A) are from the same animals shown in Figures $3 \mathrm{G}$ and $3 \mathrm{H}$.

(B and C) Resting membrane potential is hyperpolarized in 1-LNVs of alrm-GAL4>UAS$d \operatorname{TrpA1;PDF-LexA>LexAop2-CD2:\because GFP(\mathrm {B})\text {butnot}ctrl>UAS-dTrpA1;PDF-}$ LexA $>$ LexAop2-CD2: $: G F P(\mathrm{C})$ control flies at $29^{\circ} \mathrm{C}$ ("+heat"), compared to $22^{\circ} \mathrm{C}$ (RT). The data shown in panels (B and C) are from the same animals shown in Figures 3I-3K. (D and E) Cell-attached recordings of 1-LNvs, showing mean firing rate in R86E01GAL4>UAS-dTrpA1; PDF-LexA>LexAop2-CD2::GFP flies (D) and ctrl $>U A S$ dTrpA1;PDF-LexA>LexAop2-CD2::GFP controls (E), before and after thermogenetic activation from room temperature ("RT") to $28^{\circ} \mathrm{C}$ ("+heat").

\section{Figure S5. Additional behavioral and imaging data for TyrRII, Related to Figure 4 and Figure 6}

(A) Average $24 \mathrm{hr}$ sleep profiles from baseline and post-sleep deprivation days overlaid in one plot from R86E01-GAL4>ctrl (gray) and R86E01-GAL4>UAS-TyrRII-RNAi\#1 (green) flies. White and black bars indicate $12 \mathrm{hr}$ light and dark periods.

(B-E) Daytime sleep time (B), nighttime sleep time (C), nighttime sleep bout duration (D), and nighttime sleep bout number (E) under baseline conditions for R86E01GAL4>ctrl, R86E01-GAL4>UAS-TyrRII-RNAi\#1, and R86E01-GAL4>UAS-TyrRII- 
RNAi\#2 flies. Note that the data shown in panels (A-E) are from the same animals as shown in Figures 4D-4F.

(F) Average sleep profile across baseline day and sleep deprivation from $M i\{P T$ -

GFSTF.2 TyrRII $^{M I 12699}$ heterozygote and homozygote siblings suggests that the GFP tag does not disrupt TyrRII function.

$(\mathrm{G}$ and $\mathrm{H})$ Sleep recovered $(\%)(\mathrm{G})$ and daily sleep amount $(\mathrm{H})$ for heterozygous $(\mathrm{n}=23)$ and homozygous Mi\{PT-GFSTF.2\}TyrRIIIII2699 (n=26) flies.

(I) Representative images of TyrRII::GFP signal at the AL from Mi\{PT-

GFSTF.2 3 TyrRII MII2699/+ flies. Whole-mount brains were immunostained with anti-GFP (green) and anti-BRP (magenta) at ZT0-1 in the presence $(\mathrm{SD}, \mathrm{n}=5)$ or absence $(\mathrm{n}=5)$ of $12 \mathrm{hr}$ sleep deprivation.

( $\mathrm{J}$ and $\mathrm{K}$ ) Number $(\mathrm{J})$ and size $(\mathrm{K})$ of TyrRII::GFP puncta in the AL at ZT1 under baseline (ctrl) or SD conditions.

(L) Representative Airyscan super-resolution images of TyrII::GFP signal within the AL neuropil from Mi\{PT-GFSTF.2\}TyrRII ${ }^{M I 12699 /+, ~ R 86 E 01-G A L 4>U A S-m C D 4:: t d T o m a t o ~}$ immunostained with anti-GFP (green) and anti-tdTomato (magenta) at ZT0-1 in the presence (SD) or absence of $12 \mathrm{hr}$ SD. Scale bars denote $20 \mu \mathrm{m}$ in (I) and $5 \mu \mathrm{m}$ in (L).

Figure S6. Spz in astrocytes and Toll receptor in $\mathrm{R} 5$ neurons are required for sleep homeostasis, but not baseline sleep, Related to Figure 7

(A) Average $24 \mathrm{hr}$ sleep profiles from baseline and post-sleep deprivation days overlaid in one plot from R86E01-GAL4>ctrl (gray) and R86E01-GAL4>UAS-spz-RNAi\#1 (green) flies. White and black bars indicate $12 \mathrm{hr}$ light and dark periods.

(B-E) Daytime sleep time (B) nighttime sleep time (C), nighttime sleep bout duration (D), and nighttime sleep bout number (E) under baseline conditions for R86E01GAL4>ctrl, R86E01-GAL4>UAS-spz-RNAi\#1, and R86E01-GAL4>UAS-spz-RNAi\#2

flies. Note that the data in panels (A-E) are from the same animals as in Figures 7A-7C.

(F) Relative fold change in spz mRNA level in thermogenetically activated alrmGAL4>UAS-dTrpA1 ( $\mathrm{n}=3$ replicates) vs $c t r l>U A S-d \operatorname{Trp} A 1$ ( $\mathrm{n}=3$ replicates) flies from astrocyte-TRAP (pulldown) vs whole head (input) samples. Control repo/nSyb ratios for this experiment are provided in Figure 5D. 
(G) Average $24 \mathrm{hr}$ sleep profiles from baseline and post-sleep deprivation days overlaid in one plot from R58H05-GAL4>ctrl (gray) and R58H05-GAL4>UAS-Toll-RNAi\#1 (green) flies. White and black bars indicate $12 \mathrm{hr}$ light and dark periods.

(H-K) Daytime sleep time (H), nighttime sleep time (I), nighttime sleep bout duration (J), and nighttime sleep bout number $(\mathrm{K})$ under baseline conditions for R58H05-GAL4>ctrl, R86E01-GAL4>UAS-Toll-RNAi, and R86E01-GAL4>UAS-Toll-miR flies. Note that data from panels (G-K) are from the same animals as shown in Figures 7E-7G.

Figure S7. Genetic expression patterns of QF2 lines generated in this study, Related to Figure 7

(A and B) alrm-QF2 expression recapitulates alrm-GAL4 labeling. alrm-QF2 labels astrocytes throughout the brain as demonstrated in the AL (A) and SMP (B) in alrmQF2>QUAS-mCD4::mtdTomato flies, immunostained using anti-dsRed antibodies. White arrows identify cell bodies with distinct astrocyte-like morphology. Scale bars denote $20 \mu \mathrm{m}$.

Table S1. Nomenclature, genotypes and sources for fly strains used in this study. 


\section{METHODS}

\section{Fly Strains}

Flies were maintained on standard food containing molasses, cornmeal, and yeast at room temperature. Mated female flies backcrossed at least 5 generations against the $i s o^{31}$ strain or generated in the $i s o^{31}$ background[74] were used for all experiments. See Table S1 for nomenclature, genotypes and sources for fly strains used. Unless otherwise stated, all $U A S-R N A i$ lines used in this screen were obtained from the Vienna Drosophila Resource Center (VDRC, www.vdrc.at) or the Transgenic RNAi Project (TRiP) at Harvard Medical School.

\section{Nomenclature}

Because two different groups of ellipsoid body (EB) rings neurons have been named "R2" neurons[75, 76], for clarity we have adopted the EB ring nomenclature outlined by Omoto et al.[77] and refer to the sleep drive neurons we previously characterized[36] as "R5" neurons.

\section{Molecular Biology}

The alrm-QF2 line was generated by subcloning the enhancer region into pPTQF\#7hsp70 (Addgene\# 46136) using EcoRI and BamHI. The R58H05-AD and R46C03-DBD lines were generated by subcloning their respective enhancer regions into pBPp65ADZpUw (Addgene\# 26234) or pBPZpGAL4DBDUw (Addgene\# 26233), using Gateway cloning (Thermo Fisher). The following primers were used for PCR amplification of enhancers: R58H05-F 5' - ATT ACC ATG CTG GAC CGG GTG CAA GG - 3'; R58H05-R 5' - CTC ACA AGT CAT GGC CCT AAC GAG G - 3'; alrm-F 5' GAT CGA TCG CGG CCG CTA GTG GCG ATC CTT TCG CTC G - 3'; alrm-R 5' GAT CGG TAC CGA GTT AAT ATG GTG GGA ACT GC - 3'; $R 46 C 03-F$ 5' - GAT CAA AGT TTG GGG CAA CTA CCC T - 3'; and R46C03- $R$ 5' - GGT TCC CGC AAA GTT AAT CTC CTG T - 3'. The construct for UAS-Toll-miR was generated as previously described[45]. Two 22mers bridging the 1st and 2nd coding exons of the neural transcript of the Toll gene (TCT CGA ACT AAG GGC AAA TAT C and GGC GAG GGC TAC AAC AAT AAT C) were used to create the two hairpin loops. The entire microRNA construct was 
synthesized in vitro (GeneArt) and then subcloned into pUAST using EcoRI and NotI. Transgenic lines were generated in the $i s o^{3 l}$ background either through P-element mediated random insertion (alrm-QF2 and UAS-Toll-miR) or site-directed PhiC31mediated insertion into the $86 F b(R 58 H 05-A D)$ and $v k 27(R 46 C 03-D B D)$ insertion sites (Rainbow Transgenics).

\section{Behavioral Analyses}

Baseline sleep measurements: Sleep data collection was performed as previously described[45]. All analysis was performed using custom Matlab scripts (Mathworks) according to previously established algorithms[36]. Briefly, $\mathrm{CO}_{2}$-anesthetized 3-4 day old female flies were loaded into $5 \times 65 \mathrm{~mm}$ diameter glass tubes with 5\% sucrose in agar and sealed with wax and yarn, and then allowed to recover for $\sim 1.5$ days prior to data collection. Flies were loaded into Drosophila Activity Monitoring System devices (DAMS, Trikinetics), which were placed in incubators at $22^{\circ} \mathrm{C}$ with independent lighting control (12 hr:12 hr L:D cycles). Sleep epochs were identified based on the previously established criterion of 5 contiguous min of locomotor activity quiescence[78].

Thermogenetic activation: For all thermogenetic activation experiments, $U A S$-dTrpAl on the $2^{\text {nd }}$ chromosome was used, and activation was performed by ramping the temperature of the incubator according to the schedules described herein. Continuous temperature monitoring revealed an $\sim 30 \mathrm{~min}$ lag for temperatures to stably reach desired setpoints, which is reflected in the times used for analysis of sleep during activation for the $1 \mathrm{hr}$ pulse (i.e., ZT0.5-ZT1.5).

Mechanical sleep deprivation: Flies were mechanically stimulated for 2-10 s/min from ZT12-ZT24 using a vortexer mounting plate and multi-tube vortexer (Trikinetics). Only data from flies with $\geq 90 \%$ reduction in sleep amount during deprivation, compared with baseline conditions, were included for analysis. "\% Sleep Recovered" was calculated by using a sliding 30 min window to subtract baseline sleep from post-deprivation sleep binwise and then summing each with all previous bins to provide a cumulative tally of sleep time over the twelve hours post-deprivation. We then divided each $30 \mathrm{~min}$ bin by 
the total sleep lost (nighttime sleep during baseline - nighttime sleep during deprivation) and converted this ratio to a percentage.

Arousal threshold analysis: Flies were mechanically stimulated for $1 \mathrm{~s} / \mathrm{hr}$ from ZT15ZT21 and again from ZT1-ZT7 using a vortexer mounting plate and multi-tube vortexer (Trikinetics). The intensity of stimulus was varied using the speed adjustment knob of the vortexer from 1 (mild) to 3 (moderate) to 7 (strong) with two consecutive hourly pulses at each respective intensity, in that order. These brief arousals were performed with concomitant overnight thermogenetic activation $\left(28^{\circ} \mathrm{C}\right)$ from ZT12-24 and a return to baseline temperatures from ZT0-12 $\left(22^{\circ} \mathrm{C}\right)$. Flies that were inactive for $5 \mathrm{~min}$ before a stimulus and exhibited beam crossings within $3 \mathrm{~min}$ after the mechanical stimulus were identified as "aroused" and where possible the two repeats for each animal at each intensity and temperature condition were averaged. The percentage was calculated as the number of animals aroused compared to all potentially arousable animals (i.e., inactive for 5 min prior to stimulus) and each experiment was performed in triplicate.

$\underline{\text { RNAi screens: }}$ For the $\mathrm{Ca}^{2+}$ effector miniscreen, we identified genes encoding proteins that flux $\mathrm{Ca}^{2+}$, including ionotropic receptors, channels, transporters, and exchangers. $U A S-R N A i$ lines for these genes were crossed to $R 86 E 01-G A L 4$, and the appropriate progeny $(\mathrm{n}=8)$ were assessed for sleep rebound phenotypes following mechanical SD from ZT12-ZT24, as described above. For our large-scale screen 3,200 UAS-RNAi lines were selected for genes that were either randomly selected or identified from previous mammalian and invertebrate astrocyte expression studies[51, 79]. These UAS-RNAi lines were crossed to alrm-GAL4>UAS-dTrpAl flies, and the appropriate progeny $(\mathrm{n}=4)$ were assessed for "sleep rebound" phenotypes for the $6 \mathrm{hrs}$ following a $1 \mathrm{hr} 31^{\circ} \mathrm{C}$ heat pulse from ZT0-1. Lines exhibiting sleep parameters $\geq 2.5$ SD less than the mean were selected for secondary screening and further characterization.

Video analysis: Flies were loaded into 3D-printed Raspberry Pi-enabled recording chambers (Ethoscopes) and recorded using high-resolution video mode[80]. After two baseline days for acclimatization, the animals were recorded for $2 \mathrm{hrs}$ during 
thermogenetic activation (ZT 20-22, $28^{\circ} \mathrm{C}$ ) and $2 \mathrm{hrs}$ after activation (ZT 2-4, $22^{\circ} \mathrm{C}$ ). The first hour of each recording was used for manual scoring of behavior using open-source Behavioral Observation Research Interactive Software (BORIS)[81]. Behaviors were categorized into 4 possible states each minute. In cases where multiple behaviors were observed within the same minute, they were classified in this order: feeding $>$ grooming $>$ locomotion $>$ immobility such that only the highest order state was labelled regardless of the presence of the other behavioral states.

\section{Imaging}

Confocal Microscopy: All confocal images were acquired from cover-slipped (size 1.5) and Vectashield (Vector Labs) mounted samples using an LSM710 microscope and Zen Black image capture software (Zeiss International), except for CaMPARI and superresolution images which were performed on an inverted LSM800 fitted with Airyscan detectors. In the latter case, Airyscan detectors were calibrated to the brightest signal from the experimental condition and then these settings were used for all samples. In all cases, pinhole aperture and slice thickness were optimized according to the software recommendations for lens NA, magnification, and reported XY resolution.

Ex vivo CaMPARI imaging: 4-5 day old flies were loaded into locomotor tubes for sleep recordings $+/$ - mechanical deprivation (as described above). Animals were removed between ZT0-ZT3 or ZT12-15 and quickly dissected in Adult Hemolymph-Like Saline (AHLS, $103 \mathrm{mM} \mathrm{NaCl}, 3 \mathrm{mM} \mathrm{KCl}, 1.5 \mathrm{mM} \mathrm{CaCl}_{2}, 4 \mathrm{mM} \mathrm{MgCl}$, $26 \mathrm{mM} \mathrm{NaHCO}_{3}, 1$ $\mathrm{mM} \mathrm{NaH} 2 \mathrm{PO}_{4}, 10 \mathrm{mM}$ trehalose, $10 \mathrm{mM}$ glucose, $5 \mathrm{mM}$ TES Buffer, $2 \mathrm{mM}$ sucrose) and then transferred to glass bottomed dishes (Pelco, 14035-20). Data were collected as $1024 \times 1024$ pixel confocal stacks targeting approximately $10 \mu \mathrm{m}$ thick slices of the Antennal Lobes (AL) and Superior Medial Lobes (SMP) centered on astrocyte cell bodies using a 40X lens (Zeiss Plan-Apochromat 63x/1.4 Oil) prior to, and immediately after, photoconversion (PC) using 20\% intensity light generated by a TTL LED (Excite) filtered with a 395/25 nm bandpass filter. Photoconversion was achieved by quickly cycling the LED with a $500 \mathrm{~ms} / 200 \mathrm{~ms}$ duty cycle performed for 240 cycles $(\sim 2.8$ minutes total). Data were analyzed by first applying the "TurboReg" plugin of ImageJ (NIH) to 
align pre- and post-PC images and then manually drawing ROIs covering individual astrocyte cell bodies and a single neuropil region using the green channel of pre-PC images. Data analyses and generation of heat-mapped images were performed with a custom macro written for ImageJ (.ijm file available upon request) by first performing maximal intensity projection and then applying the photoconversion algorithm used by Moeyaert et al., 2018[34]. Briefly, "Fold R/G" for images represent (Red/Green)post divided by (Red/Green)pre at the pixel level. Average Fold R/G across each ROI was used for quantification.

Immunocytochemistry: Immunostaining of whole-mount brains was performed as previously described[36]. Briefly, brains were fixed in 4\% PFA for $\sim 30 \mathrm{~min}$, washed 5x in PBST (PBS + 0.3\% Triton X-100), then blocked in normal goat serum, before incubation with rabbit anti-GFP (Invitrogen, 1:1000), chicken anti-GFP (Invitrogen, 1:200), rabbit anti-DsRed (Clontech, 1:1000), or mouse anti-brp (nc82, Development Studies Hybridoma Bank, 1:20), at $4^{\circ} \mathrm{C}$ for $\sim 48 \mathrm{hrs}$, followed by incubation with Alexa 488 anti-rabbit (Invitrogen, 1:1000), Alexa 488 anti-chicken (Invitrogen, 1:1000), or Alexa 568 anti-mouse (Invitrogen, 1:1000) secondary antibodies at $4^{\circ} \mathrm{C}$ for $2-24 \mathrm{hrs}$.

TyrRII::GFP quantification: Data were collected from flies expressing GFP-tagged Tyramine Receptor II as 1024x1024 pixel confocal stacks using a 40x lens (Zeiss PlanApochromat 40x/1.3 Oil) and were cropped to include the AL. Stacks were processed and calculated using a custom macro written for ImageJ using "Maximum Entropy Thresholding" and the "Analyze Particles" function with a 3-100 pixel cutoff to quantify GFP puncta (.ijm file available upon request).

In vivo astrocyte GCaMP imaging: 5-6 day old flies were anesthetized on ice and placed in a hole that was etched on a stainless steel shim attached to a custom 3D-printed holder. The head capsule and thorax were glued to the shim using UV cured glue (Loctite, 3972). Legs, proboscis and antennae were immobilized using beeswax applied with a heated metal probe. The head capsule was bathed in AHLS. A small window was opened by removing the cuticle above the central brain using sharpened forceps (Dumont 5SF). Fat 
and other tissue were removed to gain optical access to the brain. Astrocytes expressing myristoylated GCaMP6s were imaged using 2-photon microscopy from ZT0-2 with a Zeiss LSM 710 microscope using a Ti:Sapphire Laser (@920nm, Chameleon Ultra II, Coherent). Images were acquired at 0.484 seconds a frame $(\sim 2.1 \mathrm{~Hz})$. The image window was $80 \times 80 \mu \mathrm{m}$ at $256 \times 256$ pixel resolution, and duration of imaging did not exceed 8 min per animal. The imaging plane was limited to the superior medial protocerebrum (SMP). Animals exhibiting spontaneous astrocytic $\mathrm{Ca}^{2+}$ activity were analyzed. Acquired images were first motion-corrected using a previously published method[82] with custom parameters. Motion-corrected images were processed using AQuA (Astrocyte Quantitative Analysis) which allows characterization of spatiotemporally distinct events[83]. Events were calculated based on empirically determined parameters, which were used for all images. Traces from each event were exported and filtered with a $3^{\text {rd }}$ order Savitzky-Golay filter over 15 frames.

\section{Ellipsoid body $R 5$ ring GCaMP imaging:}

For intracellular $\mathrm{Ca}^{2+}$ measurements of the R5 ellipsoid body ring, 5-6 day old $\mathrm{R} 58 \mathrm{HO5}$ QF2>QUAS-GCAMP6s, QUAS-mtdTomato-3xHA flies bearing either alrm-GAL4>UAS$d T r p A 1$ or $U A S-d T r p A 1$ alone transgenes were examined. All animals were administered a heat stimulus $\left(31^{\circ} \mathrm{C}\right)$ from ZT0-ZT1 and dissected and imaged from ZT3-ZT5. Brains were quickly dissected in AHLS and imaged in the same solution using an Ultima multiphoton microscope (Prairie Technologies). Excitation of both GCaMP6s and mtdTomato was achieved with 920nm light produced by a Ti:Sapphire Laser (Chameleon Ultra II, Coherent). Data were collected using a 40x water immersion lens with 2x optical detector zoom (Olympus LUMPLFLN 40XW/0.8) as a single 512x512 pixel plane over $60 \mathrm{~s}$ at a frequency of $\sim 1 \mathrm{~Hz}$ using Prairieview software (Prairie Technologies). The imaging plane was selected based on the completeness of the R5 ring which sits almost perpendicular to the dorsal surface of the fly brain. Data were analyzed using ImageJ by calculating the mean intensity of the R5 ring targeting ROIs (after pixel by pixel GCaMP/mtdTomato normalization) averaged over the full recording. 


\section{Electrophysiology}

3 to 9 day old flies were used in whole-cell patch clamp and cell-attached recordings. Whole-cell patch clamp recordings were generally performed as described previously[45]. For sleep deprivation experiments, flies were dissected from ZT0-2 following either $12 \mathrm{hrs}$ baseline sleep or $12 \mathrm{hrs}$ of mechanical SD (from ZT12-ZT24) as described above or by using the ethoscope tracking system[80], and whole-cell patch clamp recordings were performed using current-clamp mode. For astrocyte activation experiments, either whole-cell or cell-attached patch-clamp recordings were performed using current-clamp mode. Brains were dissected and recordings were performed in a Drosophila physiological saline solution $\left(101 \mathrm{mM} \mathrm{NaCl}, 3 \mathrm{mM} \mathrm{KCl}, 1 \mathrm{mM} \mathrm{CaCl}_{2}, 4 \mathrm{mM}\right.$ $\mathrm{MgCl}_{2}, 1.25 \mathrm{mM} \mathrm{NaH}_{2} \mathrm{PO}_{4}, 20.7 \mathrm{mM} \mathrm{NaHCO}_{3}$, and $5 \mathrm{mM}$ glucose [pH 7.2]), prebubbled with $95 \% \mathrm{O}_{2}$ and $5 \% \mathrm{CO}_{2}$, at room temperature. If needed for removal of the glial sheath, brains were treated with $2 \mathrm{mg} / \mathrm{ml}$ protease XIV (Sigma-Aldrich) for 5-8 min at $22^{\circ} \mathrm{C}$. For both whole-cell and cell-attached recordings, 1-LNvs were located using GFP fluorescence or infrared-differential interference contrast (IR-DIC) optics, under a fixed-stage upright microscope (BX51WI; Olympus or SliceScope, Scientifica). Patchpipettes (5-13 M $\Omega$ ) were made from borosilicate glass capillary with a Flaming- Brown puller (P-1000 or P-97; Sutter Instrument) and polished with a microforge. For wholecell recordings, the pipette was filled with internal solution containing $102 \mathrm{mM}$ potassium gluconate, $0.085 \mathrm{mM} \mathrm{CaCl}_{2}, 0.94 \mathrm{mM}$ EGTA, $8.5 \mathrm{mM}$ HEPES, $17 \mathrm{mM} \mathrm{NaCl}$ (pH 7.2), $4 \mathrm{mM} \mathrm{Mg-ATP,} \mathrm{and} \mathrm{0.5} \mathrm{mM} \mathrm{Na-GTP.} \mathrm{For} \mathrm{cell-attached} \mathrm{recordings,} \mathrm{the} \mathrm{pipette}$ was filled with the filtered Drosophila physiological saline solution. For astrocyte dTrpA1 activation experiments heat stimulation was applied by perfusing solution that was preheated using a temperature controller (ThermoClamp-01, Automate Scientific, Berkeley, CA or Scientifica Temperature Controller, Scientifica) into the recording chamber. Recordings were acquired with an Axopatch 200B or Multiclamp 700B amplifier (Molecular Devices) and Digidata 1440A or Digidata 1550B interface (Molecular Devices), using pCLAMP 10 or 11 . Signals were sampled at 10 or $20 \mathrm{kHz}$ and low-pass filtered at 2 or $3 \mathrm{kHz}$. For each cell-attached recording, a brief electrical stimulus (15V amplitude, 10k Hz square wave, $0.05 \mathrm{~ms}$ duration) was applied after current-clamp recordings from each cell to verify access to the cell. The cell was 
discarded if this stimulus did not elicit action potentials. For the cell-attached recordings, 2 cells with a mean firing rate $>9 \mathrm{~Hz}$ were excluded, due to concerns about cell health.

\section{Translating ribosomal affinity purification with quantitative polymerase chain reaction}

Translating ribosomal affinity purification (TRAP) was performed as previously described using purified EGFP antibody (19C8 antibody, Memorial Sloan Kettering Cancer Center)[84] to pulldown ribosomal complexes and their associated transcripts. Briefly, $\sim 1,024$ fly heads (per group) were collected in liquid $\mathrm{N}_{2}$ from 5-6 day old R86E01-GAL4>UAS-Rpl10a::EGFP flies at ZT0-2 under baseline conditions or immediately following $12 \mathrm{hrs}$ mechanical SD from ZT12-ZT24 (described above). Following short-term storage at $-80^{\circ} \mathrm{C}$, heads were pulverized in homogenization buffer and then incubated with antibody-coupled magnetic beads (Dynabeads Antibody Coupling Kit, Invitrogen) to immunoprecipitate ribosomal bound mRNA species. After RNA extraction (Trizol, Invitrogen), cDNA libraries were synthesized using SuperScript III high capacity Reverse Transcription Kit (Invitrogen), and qPCR was performed using the Power SYBR Green PCR master mix (Life Technologies) using the following primers: repo- $F$ 5'- GCA TCA AGA AGA AGA AGA CGA GA - 3'; repo- $R$ 5'- GTT CAA AGG CAC GCT CCA - 3'; $n S y b-F$ 5'- GGT CGA TGA GGT CGT GGA C - 3'; $n S y b-R$ 5'- CCA GAA TTT CCT CTT GAG CTT GC -3'; Rpl49-F 5'- CGG ATC GAT ATG CTA AGC TGT - 3'; Rpl49-R 5'- CGA CGC ACT CTG TTG TCG - 3'; spz-F 5'GCA ACG ATC TTC AGC CCA CG - 3'; spz-R 5' - TTGATCCGCTCCTTCGCACT 3'; TyrRII-F 5' - GGC TGG ATA CTG TGC GAC AT - 3'; TyrRII-R 5'- GTG ACG GCG AGA TAC CTG TC - 3'. A similar protocol was followed for the thermogenetic activation experiments instead using alrm-QF2>QUAS-dTrpA1; R86E01-GAL4>UASRpl10a::EGFP or ctrl>QUAS-dTrpA1; R86E01-GAL4>UAS-Rpl10a::EGFP at ZT0-2 immediately following overnight (ZT12-24) heat pulse at $28^{\circ} \mathrm{C}$.

\section{Statistical Analysis}

All analyses were performed using Prism 7 (Graphpad). Normally distributed data were analyzed using parametric tests (Student's t tests and one-way or two-way ANOVAs 
followed by Tukey's post-hoc test) and plotted as bar graphs \pm SEM, whereas data that violated the assumption of normality were analyzed using non-parametric tests (KruskalWallis test followed by Dunn's post-hoc test) and plotted as simplified boxplots (Median with $1^{\text {st }}$, and $3^{\text {rd }}$ Quartile boxes). For the video analysis, a Chi-squared analysis was performed to assess differences in the distribution of experimental alrm $>d \operatorname{Trp} A 1$ group relative to expected values provided by the $U A S$-dTrpAlcontrols. 


\section{REFERENCES}

1. Blanchard, S., and Bronzino, J.D. (2012). Anatomy and physiology. In Introduction to Biomedical Engineering (Third Edition), J.D. Enderle and J.D. Bronzino, eds. (Boston: Academic Press), pp. 75-132.

2. Reichert, S., Pavón Arocas, O., and Rihel, J. (2019). The neuropeptide galanin Is required for homeostatic rebound sleep following increased neuronal activity. Neuron 104, 370-384.e375.

3. Cirelli, C., and Tononi, G. (2019). Linking the need to sleep with synaptic function. Science. 366, 189-190.

4. Huber, R., Ghilardi, M.F., Massimini, M., and Tononi, G. (2004). Local sleep and learning. Nature. 430, 78-81.

5. Rector, D.M., Schei, J.L., Van Dongen, H.P., Belenky, G., and Krueger, J.M. (2009). Physiological markers of local sleep. The European journal of neuroscience 29, 1771-1778.

6. Borbely, A.A., and Achermann, P. (1992). Concepts and models of sleep regulation: an overview. J Sleep Res 1, 63-79.

7. Garcia-Marin, V., Garcia-Lopez, P., and Freire, M. (2007). Cajal's contributions to glia research. Trends Neurosci 30, 479-487.

8. Halassa, M.M., Florian, C., Fellin, T., Munoz, J.R., Lee, S.Y., Abel, T., Haydon, P.G., and Frank, M.G. (2009). Astrocytic modulation of sleep homeostasis and cognitive consequences of sleep loss. Neuron 61, 213-219.

9. Pelluru, D., Konadhode, R.R., Bhat, N.R., and Shiromani, P.J. (2016). Optogenetic stimulation of astrocytes in the posterior hypothalamus increases sleep at night in C57BL/6J mice. The European journal of neuroscience 43, 12981306.

10. Poskanzer, K.E., and Yuste, R. (2016). Astrocytes regulate cortical state switching in vivo. Proc Natl Acad Sci U S A 113, E2675-2684.

11. Clasadonte, J., Scemes, E., Wang, Z., Boison, D., and Haydon, P.G. (2017). Connexin 43-mediated astroglial metabolic networks contribute to the regulation of the sleep-wake cycle. Neuron 95, 1365-1380 e1365.

12. Dani, J.W., Chernjavsky, A., and Smith, S.J. (1992). Neuronal activity triggers calcium waves in hippocampal astrocyte networks. Neuron 8, 429-440.

13. Agulhon, C., Petravicz, J., McMullen, A.B., Sweger, E.J., Minton, S.K., Taves, S.R., Casper, K.B., Fiacco, T.A., and McCarthy, K.D. (2008). What is the role of astrocyte calcium in neurophysiology? Neuron 59, 932-946.

14. Papouin, T., Dunphy, J.M., Tolman, M., Dineley, K.T., and Haydon, P.G. (2017). Septal cholinergic neuromodulation tunes the astrocyte-dependent gating of hippocampal NMDA receptors to wakefulness. Neuron 94, 840-854 e847.

15. Durkee, C.A., and Araque, A. (2019). Diversity and specificity of astrocyteneuron communication. Neuroscience 396, 73-78.

16. Berridge, M.J., Lipp, P., and Bootman, M.D. (2000). The versatility and universality of calcium signalling. Nat Rev Mol Cell Biol 1, 11-21.

17. Shigetomi, E., Patel, S., and Khakh, B.S. (2016). Probing the complexities of astrocyte calcium signaling. Trends Cell Biol 26, 300-312. 
18. Bazargani, N., and Attwell, D. (2016). Astrocyte calcium signaling: the third wave. Nat Neurosci 19, 182-189.

19. Verkhratsky, A., Orkand, R.K., and Kettenmann, H. (1998). Glial calcium: homeostasis and signaling function. Physiol Rev 78, 99-141.

20. Fiacco, T.A., and McCarthy, K.D. (2006). Astrocyte calcium elevations: properties, propagation, and effects on brain signaling. Glia 54, 676-690.

21. Verkhratsky, A., and Nedergaard, M. (2018). Physiology of astroglia. Physiol Rev 98, 239-389.

22. Covelo, A., and Araque, A. (2018). Neuronal activity determines distinct gliotransmitter release from a single astrocyte. Elife 7, e32237.

23. Frank, M.G. (2013). Astroglial regulation of sleep homeostasis. Curr Opin Neurobiol 23, 812-818.

24. Freeman, M.R. (2015). Drosophila central nervous system glia. Cold Spring Harb Perspect Biol 7.

25. Vorster, A.P., and Born, J. (2015). Sleep and memory in mammals, birds and invertebrates. Neuroscience \& Biobehavioral Reviews 50, 103-119.

26. Allada, R., and Siegel, J.M. (2008). Unearthing the phylogenetic roots of sleep. Curr Biol 18, R670-R679.

27. Vyazovskiy, V.V., Olcese, U., Lazimy, Y.M., Faraguna, U., Esser, S.K., Williams, J.C., Cirelli, C., and Tononi, G. (2009). Cortical firing and sleep homeostasis. Neuron 63, 865-878.

28. Ding, F., O’Donnell, J., Xu, Q., Kang, N., Goldman, N., and Nedergaard, M. (2016). Changes in the composition of brain interstitial ions control the sleepwake cycle. Science. 352, 550-555.

29. Areal, C.C., Warby, S.C., and Mongrain, V. (2017). Sleep loss and structural plasticity. Current Opinion in Neurobiology 44, 1-7.

30. Niethard, N., and Born, J. (2019). Back to baseline: sleep recalibrates synapses. Nat. Neurosci. 22, 149-151.

31. Thomas, C.W., Guillaumin, M.C.C., McKillop, L.E., Achermann, P., and Vyazovskiy, V.V. (2019). Cortical neuronal activity determines the dynamics of local sleep homeostasis. bioRxiv, 756270.

32. Halassa, M.M., and Haydon, P.G. (2010). Integrated brain circuits: astrocytic networks modulate neuronal activity and behavior. Annu Rev Physiol 72, 335355.

33. Losi, G., Mariotti, L., Sessolo, M., and Carmignoto, G. (2017). New tools to study astrocyte $\mathrm{Ca}(2+)$ signal dynamics in brain networks in vivo. Front Cell Neurosci $11,134$.

34. Moeyaert, B., Holt, G., Madangopal, R., Perez-Alvarez, A., Fearey, B.C., Trojanowski, N.F., Ledderose, J., Zolnik, T.A., Das, A., Patel, D., et al. (2018). Improved methods for marking active neuron populations. Nat Commun 9, 4440.

35. Donlea, J.M., Thimgan, M.S., Suzuki, Y., Gottschalk, L., and Shaw, P.J. (2011). Inducing sleep by remote control facilitates memory consolidation in Drosophila. Science. 332, 1571-1576.

36. Liu, S., Liu, Q., Tabuchi, M., and Wu, M.N. (2016). Sleep drive Is encoded by neural plastic changes in a dedicated circuit. Cell. 165, 1347-1360. 
37. Rechtschaffen, A., Bergmann, B.M., Gilliland, M.A., and Bauer, K. (1999). Effects of method, duration, and sleep stage on rebounds from sleep deprivation in the rat. Sleep 22, 11-31.

38. Shang, Y., Donelson, Nathan C., Vecsey, Christopher G., Guo, F., Rosbash, M., and Griffith, Leslie C. (2013). Short Neuropeptide F is a sleep-promoting inhibitory modulator. Neuron 80, 171-183.

39. Cavanaugh, D.J., Vigderman, A.S., Dean, T., Garbe, D.S., and Sehgal, A. (2016). The drosophila circadian clock gates sleep through time-of-day dependent modulation of sleep-promoting neurons. Sleep 39, 345-356.

40. Shang, Y., Griffith, L.C., and Rosbash, M. (2008). Light-arousal and circadian photoreception circuits intersect at the large PDF cells of the Drosophila brain. Proceedings of the National Academy of Sciences 105, 19587-19594.

41. Parisky, K.M., Agosto, J., Pulver, S.R., Shang, Y., Kuklin, E., Hodge, J.J.L., Kang, K., Liu, X., Garrity, P.A., Rosbash, M., et al. (2008). PDF cells are a GABA-responsive wake-promoting component of the Drosophila sleep circuit. Neuron 60, 672-682.

42. Sheeba, V., Fogle, K.J., Kaneko, M., Rashid, S., Chou, Y.T., Sharma, V.K., and Holmes, T.C. (2008). Large ventral lateral neurons modulate arousal and sleep in Drosophila. Curr Biol 18, 1537-1545.

43. Cao, G., and Nitabach, M.N. (2008). Circadian control of membrane excitability in Drosophila melanogaster lateral ventral clock neurons. J Neurosci 28, 64936501.

44. Sheeba, V., Gu, H., Sharma, V.K., O'Dowd, D.K., and Holmes, T.C. (2008). Circadian- and light-dependent regulation of resting membrane potential and spontaneous action potential firing of Drosophila circadian pacemaker neurons. J Neurophysiol 99, 976-988.

45. Liu, S., Lamaze, A., Liu, Q., Tabuchi, M., Yang, Y., Fowler, M., Bharadwaj, R., Zhang, J., Bedont, J., Blackshaw, S., et al. (2014). WIDE AWAKE mediates the circadian timing of sleep onset. Neuron 82, 151-166.

46. Saper, C.B., Fuller, P.M., Pedersen, N.P., Lu, J., and Scammell, T.E. (2010). Sleep state switching. Neuron 68, 1023-1042.

47. Sehgal, A., and Mignot, E. (2011). Genetics of sleep and sleep disorders. Cell. 146, 194-207.

48. Weber, F., and Dan, Y. (2016). Circuit-based interrogation of sleep control. Nature. 538, 51-59.

49. Aston-Jones, G., and Bloom, F. (1981). Activity of norepinephrine-containing locus coeruleus neurons in behaving rats anticipates fluctuations in the sleepwaking cycle. J. Neurosci. 1, 876-886.

50. Weinberg, Z.Y., and Puthenveedu, M.A. (2019). Regulation of G protein-coupled receptor signaling by plasma membrane organization and endocytosis. Traffic 20, 121-129.

51. Ng, F.S., Sengupta, S., Huang, Y., Yu, A.M., You, S., Roberts, M.A., Iyer, L.K., Yang, Y., and Jackson, F.R. (2016). TRAP-seq profiling and RNAi-based genetic screens identify conserved glial genes required for adult Drosophila behavior. Front Mol Neurosci 9, 146. 
52. Nagarkar-Jaiswal, S., DeLuca, S.Z., Lee, P.T., Lin, W.W., Pan, H., Zuo, Z., Lv, J., Spradling, A.C., and Bellen, H.J. (2015). A genetic toolkit for tagging intronic MiMIC containing genes. Elife 4, e08469.

53. Ma, Z., Stork, T., Bergles, D.E., and Freeman, M.R. (2016). Neuromodulators signal through astrocytes to alter neural circuit activity and behaviour. Nature. 539, 428-432.

54. Bazargani, N., and Attwell, D. (2017). Amines, astrocytes, and arousal. Neuron 94, 228-231.

55. Krueger, J.M., Nguyen, J.T., Dykstra-Aiello, C.J., and Taishi, P. (2019). Local sleep. Sleep Med Rev 43, 14-21.

56. Li, G., Forero, M.G., Wentzell, J.S., Durmus, I., Wolf, R., Anthoney, N.C., Parker, M., Jiang, R., Hasenauer, J., Strausfeld, N.J., et al. (2020). A Toll-receptor map underlies structural brain plasticity. eLife 9, e52743.

57. Letellier, M., Park, Y.K., Chater, T.E., Chipman, P.H., Gautam, S.G., OshimaTakago, T., and Goda, Y. (2016). Astrocytes regulate heterogeneity of presynaptic strengths in hippocampal networks. Proceedings of the National Academy of Sciences 113, E2685-E2694.

58. Barres, B., Chun, L., and Corey, D. (1989). Calcium current in cortical astrocytes: induction by cAMP and neurotransmitters and permissive effect of serum factors. The Journal of Neuroscience 9, 3169-3175.

59. MacVicar, B.A. (1984). Voltage-dependent calcium channels in glial cells. Science. 226, 1345-1347.

60. Rungta, R.L., Bernier, L.-P., Dissing-Olesen, L., Groten, C.J., LeDue, J.M., Ko, R., Drissler, S., and MacVicar, B.A. (2016). Ca2+ transients in astrocyte fine processes occur via $\mathrm{Ca} 2+$ influx in the adult mouse hippocampus. Glia 64, 20932103.

61. Lipscombe, D., Helton, T.D., and Xu, W. (2004). L-Type calcium channels: the low down. Journal of Neurophysiology 92, 2633-2641.

62. Bayliss, A., Roselli, G., and Evans, P.D. (2013). A comparison of the signalling properties of two tyramine receptors from Drosophila. J Neurochem 125, 37-48.

63. O'Donnell, J., Ding, F., and Nedergaard, M. (2015). Distinct functional states of astrocytes during sleep and wakefulness: is norepinephrine the master regulator? Current Sleep Medicine Reports 1, 1-8.

64. Richter, C., Woods, I.G., and Schier, A.F. (2014). Neuropeptidergic control of sleep and wakefulness. Annu Rev Neurosci 37, 503-531.

65. Mackiewicz, M., Naidoo, N., Zimmerman, J.E., and Pack, A.I. (2008). Molecular Mechanisms of Sleep and Wakefulness. Annals of the New York Academy of Sciences 1129, 335-349.

66. Ingiosi, A.M., and Opp, M.R. (2016). Sleep and immunomodulatory responses to systemic lipopolysaccharide in mice selectively expressing interleukin-1 receptor 1 on neurons or astrocytes. Glia 64, 780-791.

67. Cajochen, C., Brunner, D.P., Krauchi, K., Graw, P., and Wirz-Justice, A. (1995). Power density in theta/alpha frequencies of the waking EEG progressively increases during sustained wakefulness. Sleep 18, 890-894. 
68. Gupta, C., López, J.M., Ott, W., Josić, K., and Bennett, M.R. (2013).

Transcriptional delay stabilizes bistable gene networks. Physical Review Letters $111,058104$.

69. Glass, L., Beuter, A., and Larocque, D. (1988). Time delays, oscillations, and chaos in physiological control systems. Mathematical Biosciences 90, 111-125.

70. Ferrell, J.E. (2002). Self-perpetuating states in signal transduction: positive feedback, double-negative feedback and bistability. Current Opinion in Cell Biology 14, 140-148.

71. Marucci, L., Barton, D.A.W., Cantone, I., Ricci, M.A., Cosma, M.P., Santini, S., di Bernardo, D., and di Bernardo, M. (2009). How to turn a genetic circuit into a synthetic tunable oscillator, or a bistable switch. PloS one 4, e8083-e8083.

72. Angeli, D., Ferrell, J.E., Jr., and Sontag, E.D. (2004). Detection of multistability, bifurcations, and hysteresis in a large class of biological positive-feedback systems. Proc Natl Acad Sci U S A 101, 1822-1827.

73. Borbély, A.A., Daan, S., Wirz-Justice, A., and Deboer, T. (2016). The twoprocess model of sleep regulation: a reappraisal. Journal of Sleep Research, n/an/a.

74. Ryder, E., Blows, F., Ashburner, M., Bautista-Llacer, R., Coulson, D., Drummond, J., Webster, J., Gubb, D., Gunton, N., Johnson, G., et al. (2004). The DrosDel collection: a set of P-element insertions for generating custom chromosomal aberrations in Drosophila melanogaster. Genetics 167, 797-813.

75. Hanesch, U., Fischbach, K.F., and Heisenberg, M. (1989). Neuronal architecture of the central complex in Drosophila melanogaster. Cell Tissue Res 257, 343366.

76. Lin, C.Y., Chuang, C.C., Hua, T.E., Chen, C.C., Dickson, B.J., Greenspan, R.J., and Chiang, A.S. (2013). A comprehensive wiring diagram of the protocerebral bridge for visual information processing in the Drosophila brain. Cell Rep 3, 1739-1753.

77. Omoto, J.J., Keles, M.F., Nguyen, B.M., Bolanos, C., Lovick, J.K., Frye, M.A., and Hartenstein, V. (2017). Visual Input to the Drosophila Central Complex by Developmentally and Functionally Distinct Neuronal Populations. Curr Biol 27, 1098-1110.

78. Shaw, P.J., Cirelli, C., Greenspan, R.J., and Tononi, G. (2000). Correlates of sleep and waking in Drosophila melanogaster. Science. 287, 1834-1837.

79. Bellesi, M., de Vivo, L., Tononi, G., and Cirelli, C. (2015). Transcriptome profiling of sleeping, waking, and sleep deprived adult heterozygous Aldh1L1 eGFP-L10a mice. Genom Data 6, 114-117.

80. Geissmann, Q., Garcia Rodriguez, L., Beckwith, E.J., French, A.S., Jamasb, A.R., and Gilestro, G.F. (2017). Ethoscopes: An open platform for high-throughput ethomics. PLOS Biology 15, e2003026.

81. Friard, O., and Gamba, M. (2016). BORIS: a free, versatile open-source eventlogging software for video/audio coding and live observations. Methods in Ecology and Evolution 7, 1325-1330.

82. Pnevmatikakis, E.A., and Giovannucci, A. (2017). NoRMCorre: An online algorithm for piecewise rigid motion correction of calcium imaging data. $\mathrm{J}$ Neurosci Methods 291, 83-94. 
83. Wang, Y., DelRosso, N.V., Vaidyanathan, T., Reitman, M., Cahill, M.K., Mi, X., Yu, G., and Poskanzer, K.E. (2018). An event-based paradigm for analyzing fluorescent astrocyte activity uncovers novel single-cell and population-level physiology. bioRxiv, 504217.

84. Heiman, M., Kulicke, R., Fenster, R.J., Greengard, P., and Heintz, N. (2014). Cell type-specific mRNA purification by translating ribosome affinity purification (TRAP). Nat Prot 9, 1282-1291. 
bioRxiv preprint doi: https://doi.org/10.1101/2020.07.04.187906; this version posted July 5, 2020. The copyright holder for this preprint (which

A
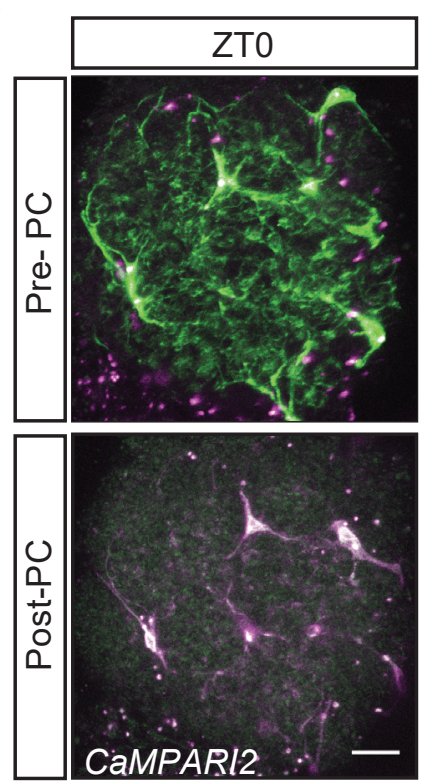

F

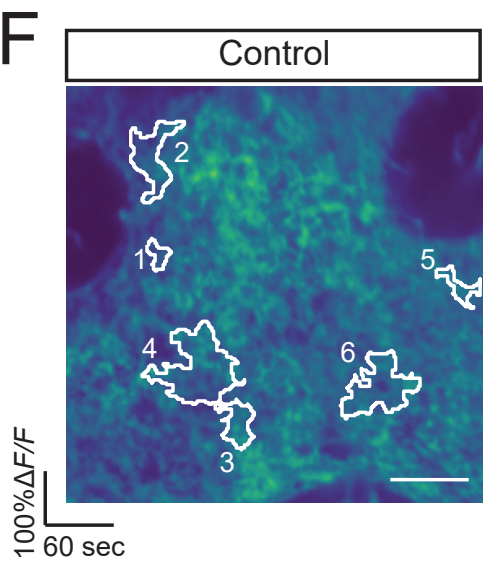

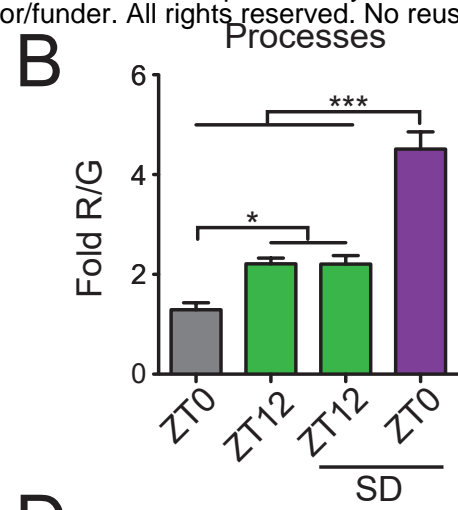
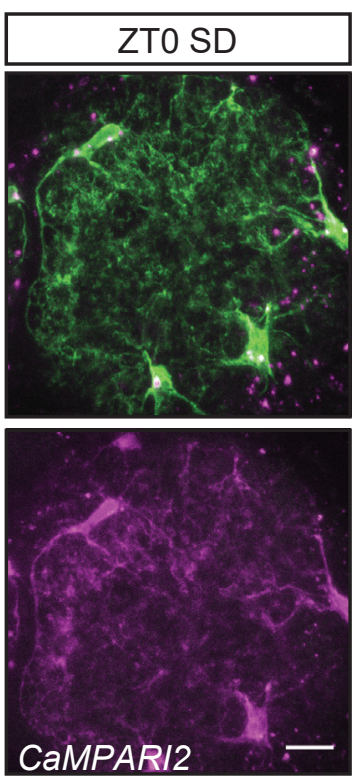

D
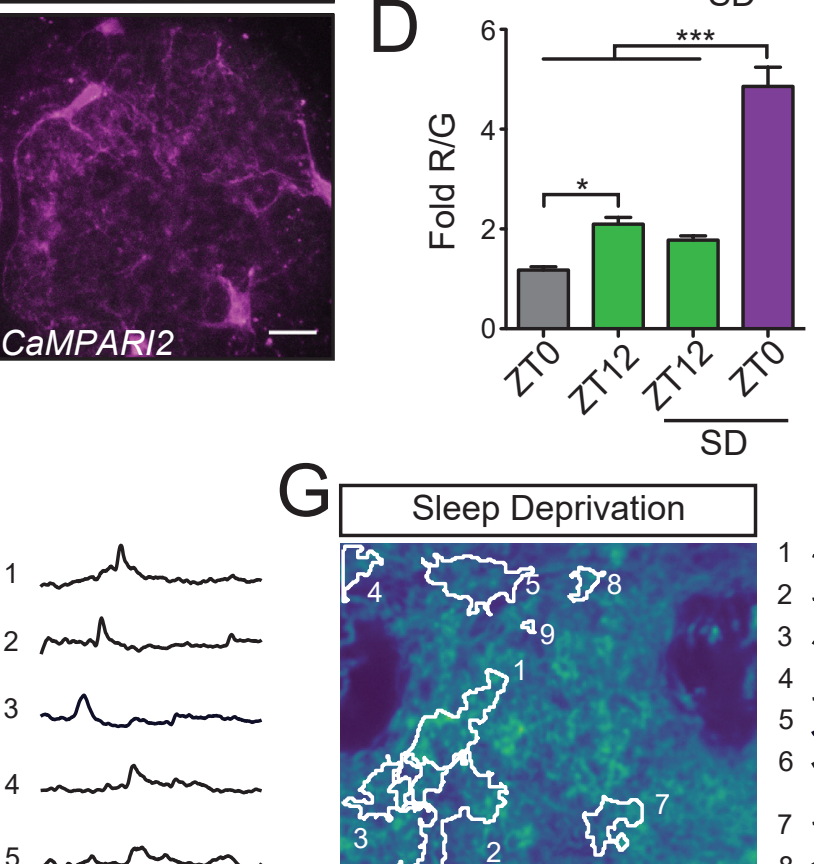

(2)

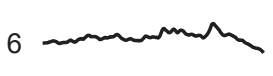

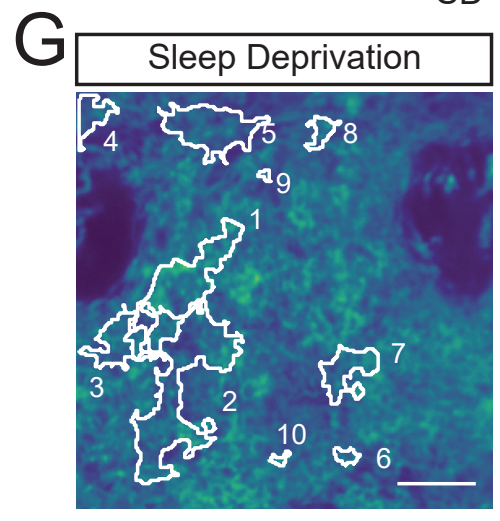

C

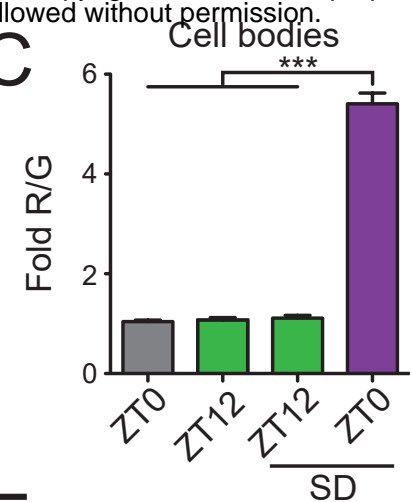

SMP

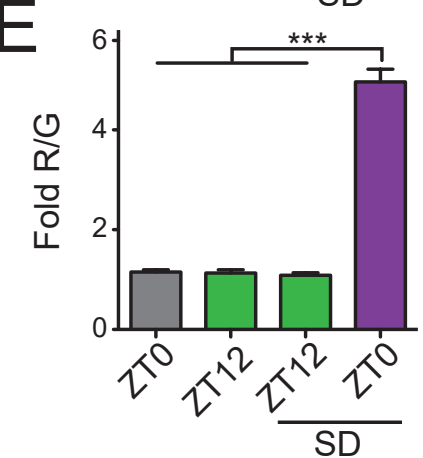

AL
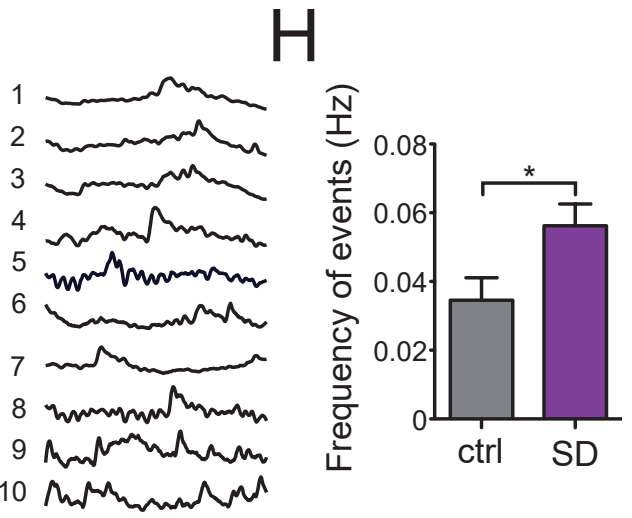
bioRxiv preprint doi: https://doi.org/10.1101/2020.07.04.187906; this version posted July 5, 2020. The copyright holder for this preprint (which was not certified by peer review) is the author/funder. All rights reserved. No reuse allowed without permission.

A was not certified by peer review) is the author/funder. All rights
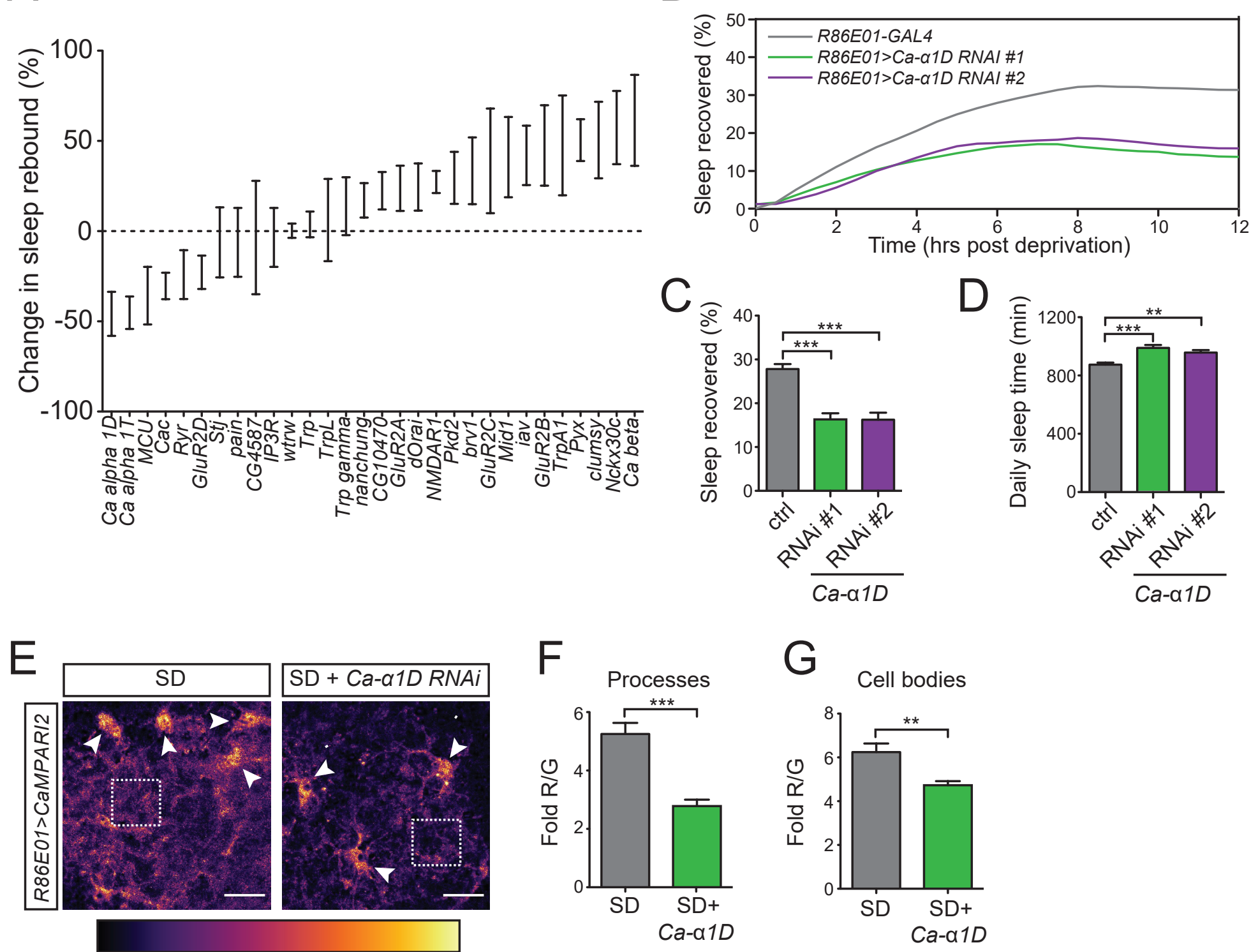
bioRxiv preprint doi: https://doi.org/10.1101/2020.07.04.187906; this version posted July 5, 2020. The copyright holder for this preprint (which was not certified by peer review) is the author/funder. All rights reserved. No reuse allowed without permission.

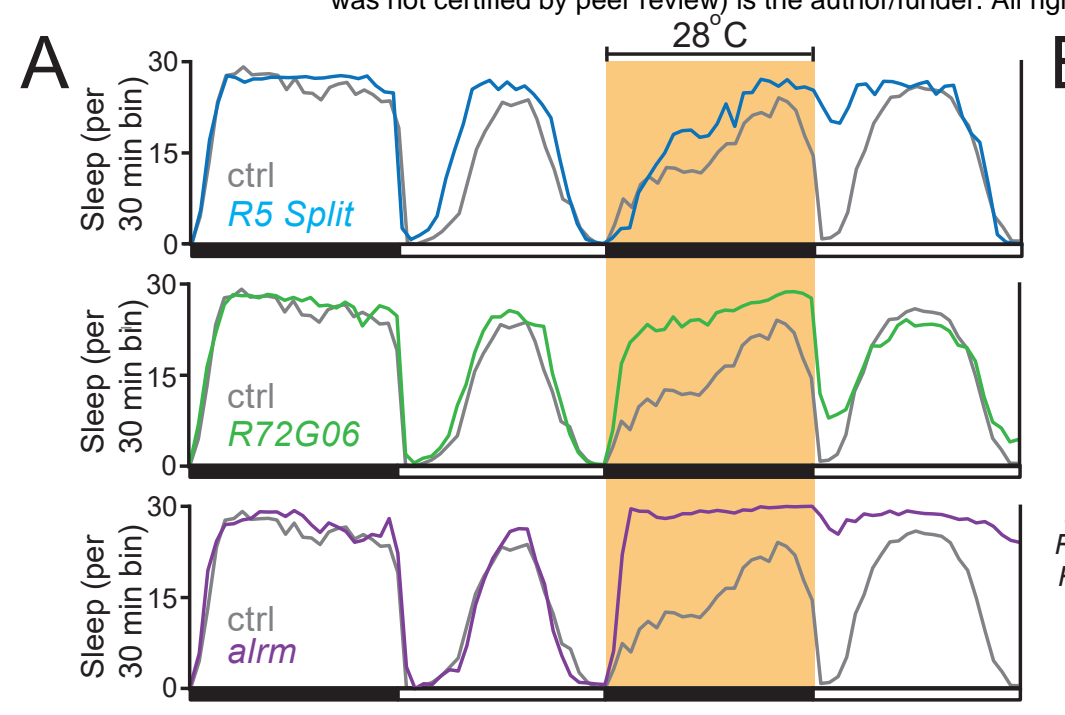

D

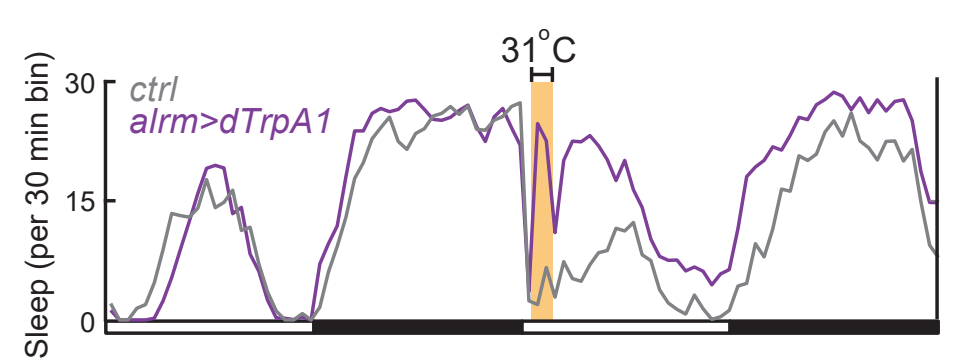

G
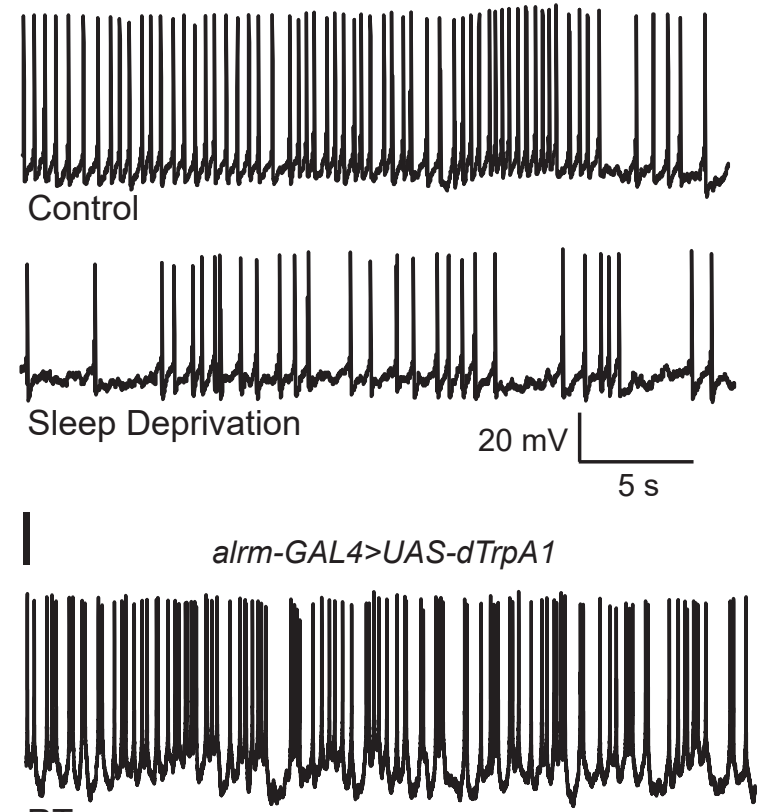

RT

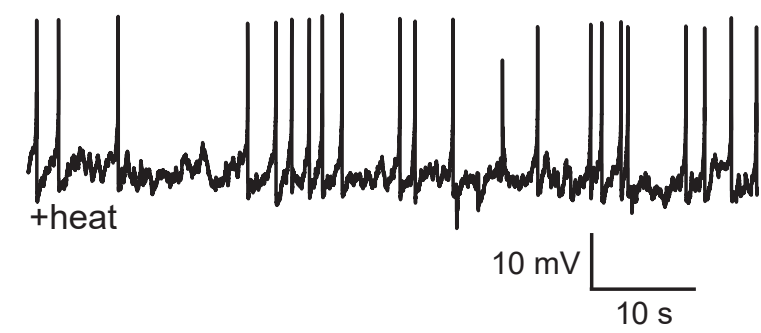

B

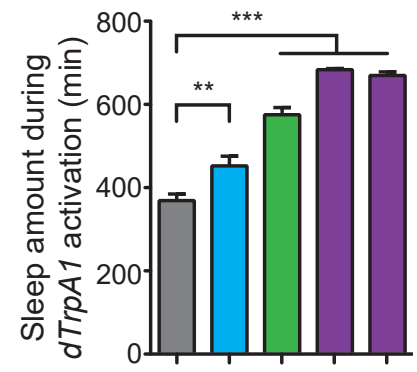

R58H05-AD;

$R 46 C 03-D B D$

R72G06-GAL4 -

R86E01-GAL4 -

alrm-GAL4 - $-2+-$

UAS-dTrpA1 + + + + +

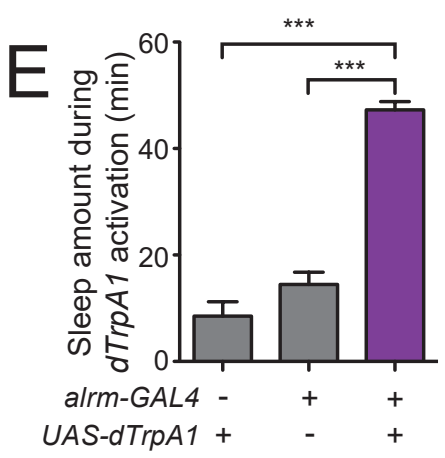

$\mathrm{H}$

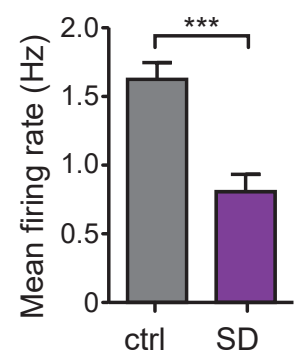

$J$

alrm-GAL4>

UAS-dTrpA1

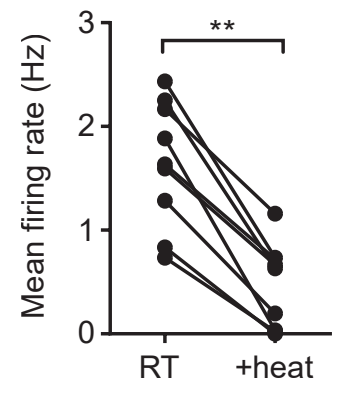

K
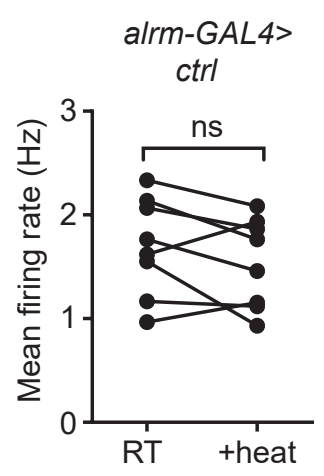

C

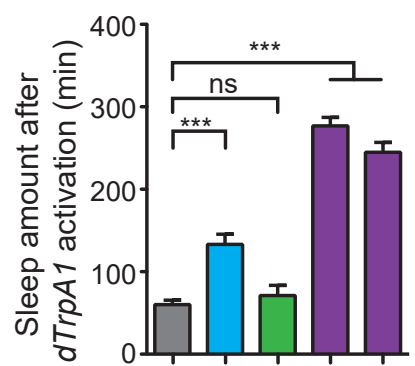

R58H05-AD;

R46C03-DBD

R72G06-GAL4

R86E01-GAL4 -

alrm-GAL4
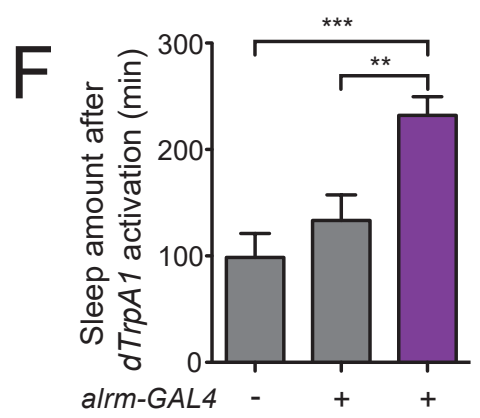

UAS-dTrpA1 + - + 
bioRxiv preprint doi: https://doi.org/10.1101/2020.07.04.187906; this version posted July 5, 2020. The copyright holder for this preprint (which
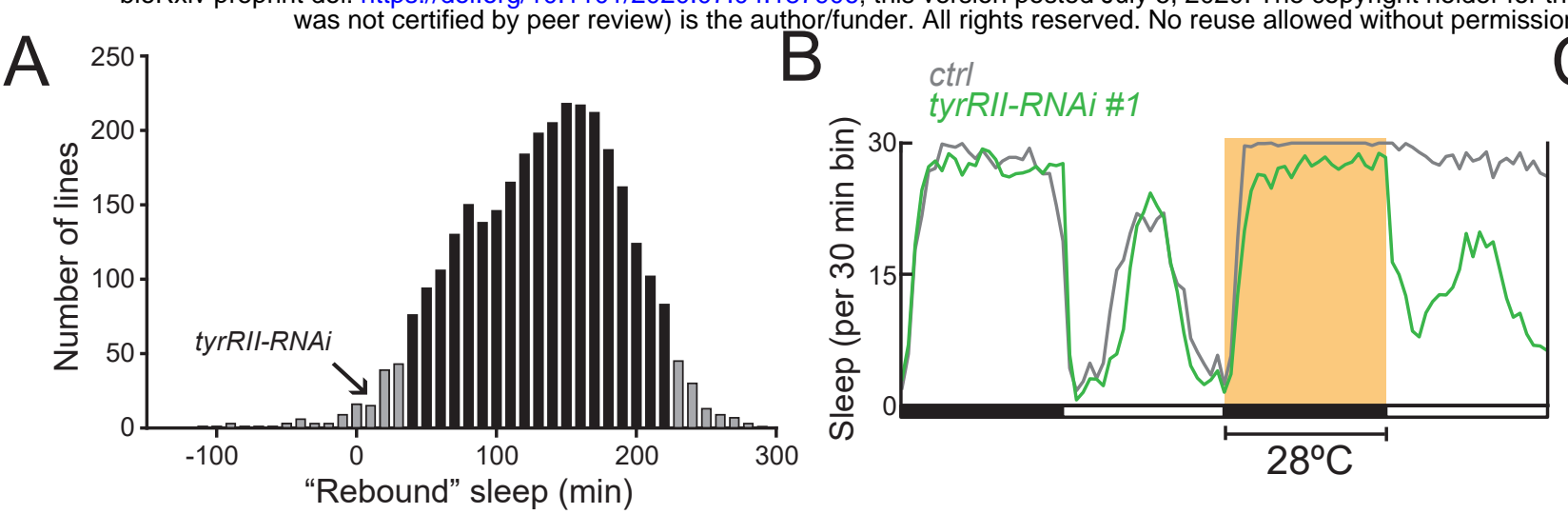

C

D

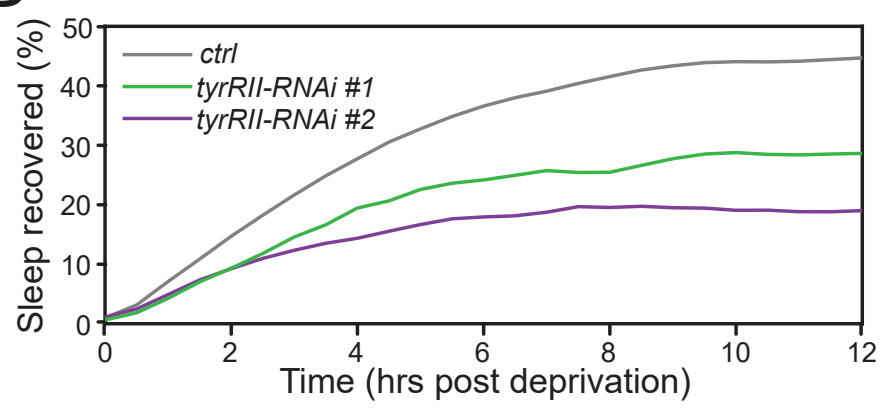

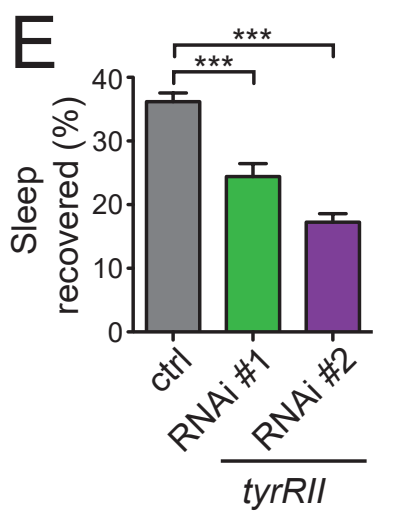
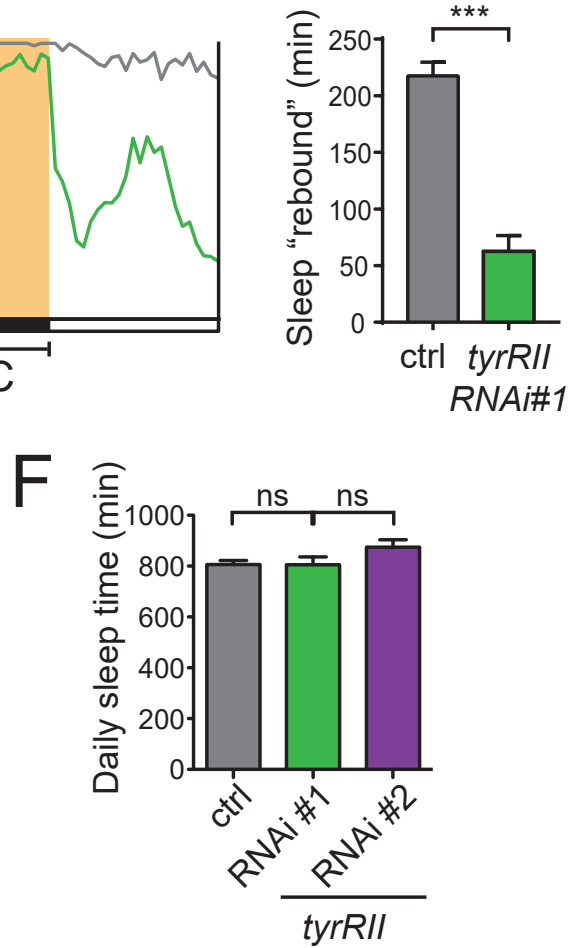
t.

A

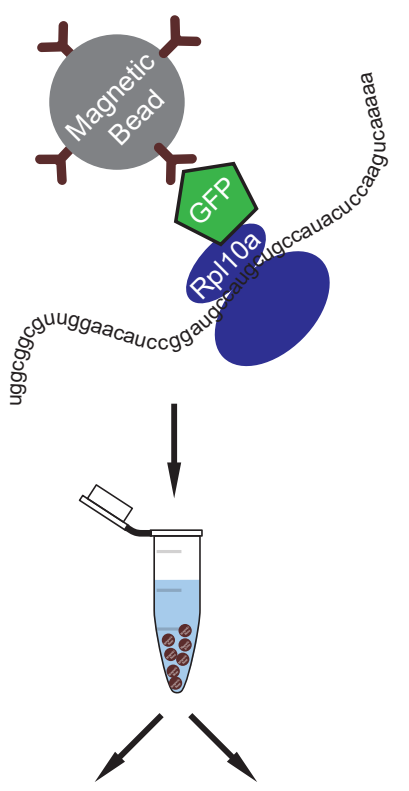

Whole Head Astrocyte Enriched Input

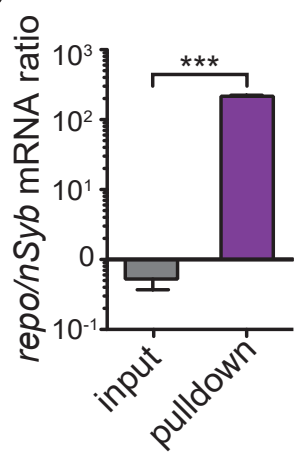

D

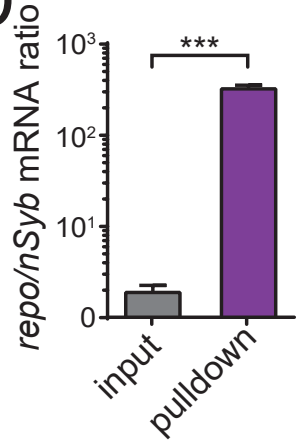

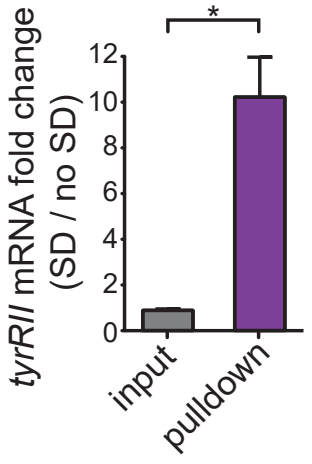

E

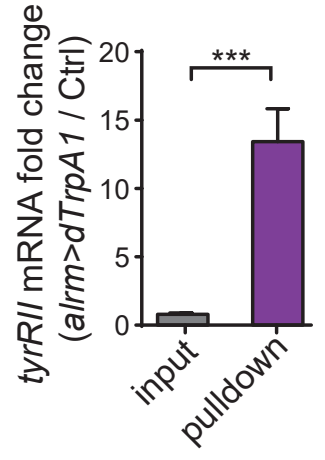


bioRxiv preprint doi: https://doi.org/10.1101/2020.07.04.187906; this version posted July 5, 2020. The copyright holder for this preprint (which was not certified by peer review) is the author/funder. All rights reserved. No reuse allowed without permission.
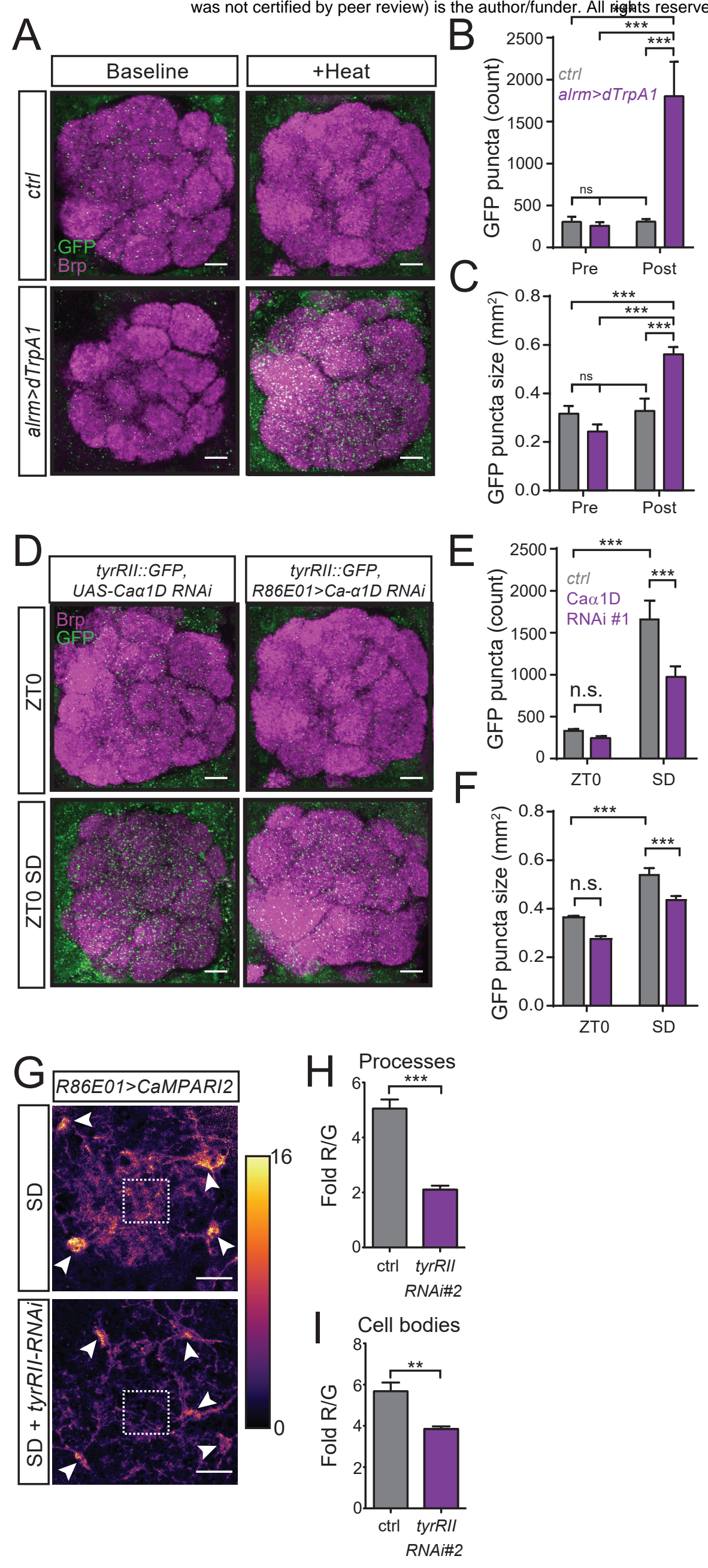
bioRxiv preprint doi: https://doi.org/10.1101/2020.07.04.187906; this version posted July 5, 2020. The copyright holder for this preprint (which

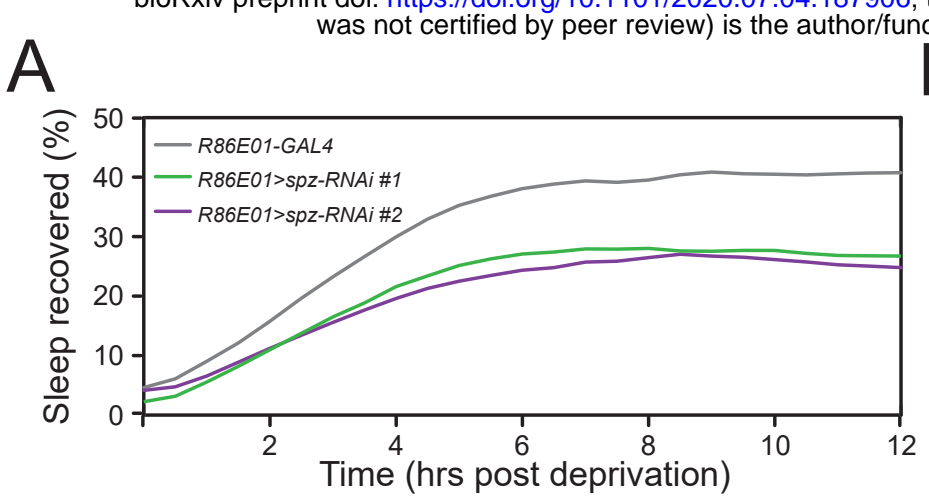

B

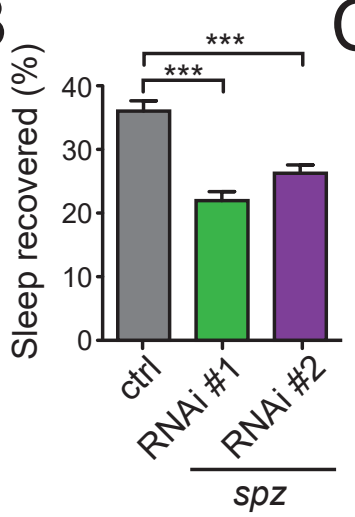

C

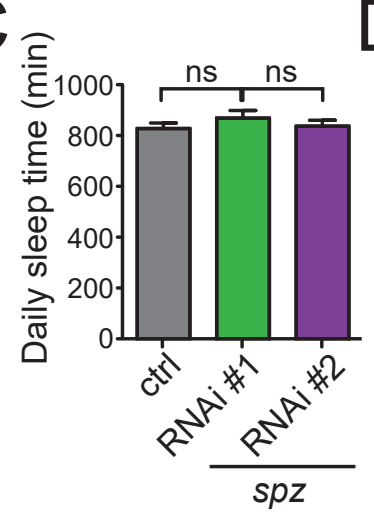

E

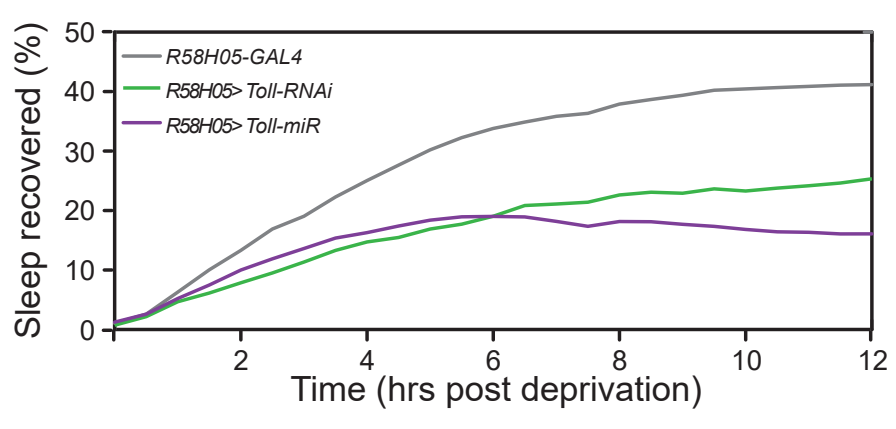

$\mathrm{F}$

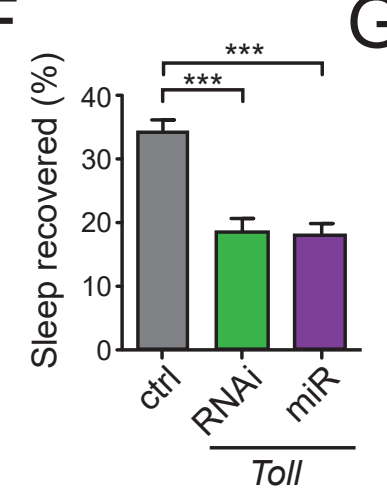

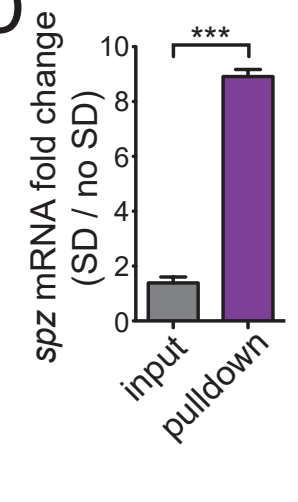

D
$\mathrm{H}$
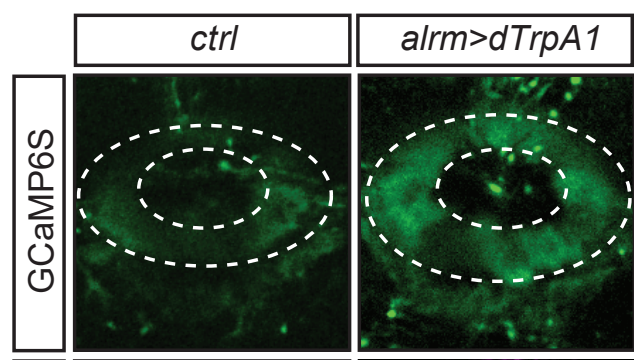

年

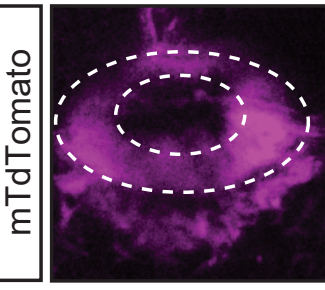

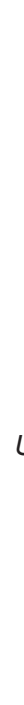

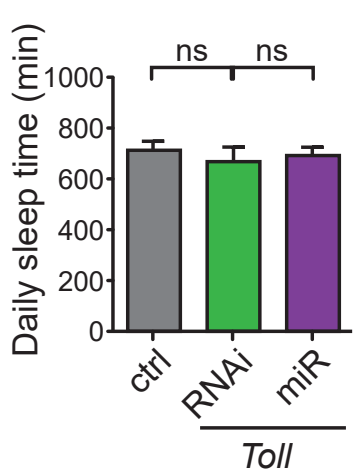

$J$

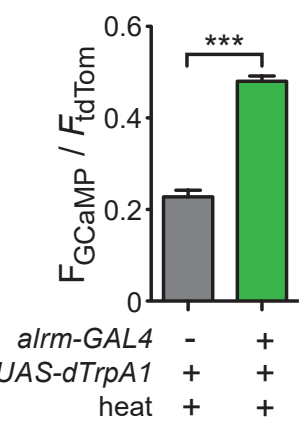

Astrocytes
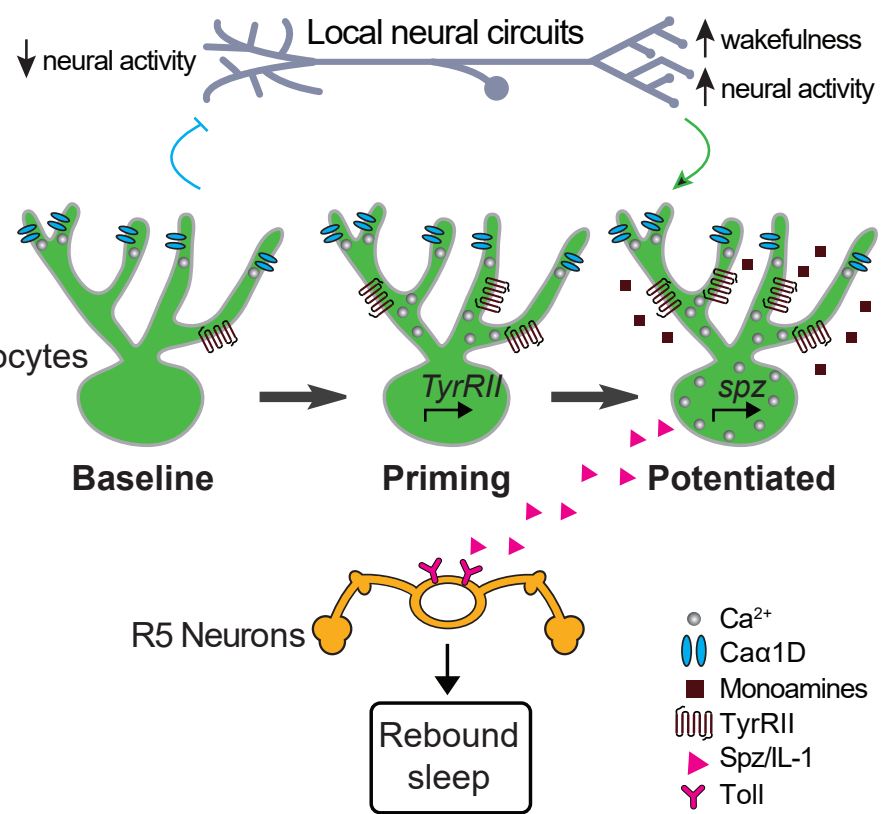

- $\mathrm{Ca}^{2+}$

00 Caa1D

- Monoamines

[nog TyrRII

- Spz/L-1

$Y$ Toll 
bioRxiv preprint doi: https://doi.org/10.1101/2020.07.04.187906; this version posted July 5, 2020. The copyright holder for this preprint (which

A was
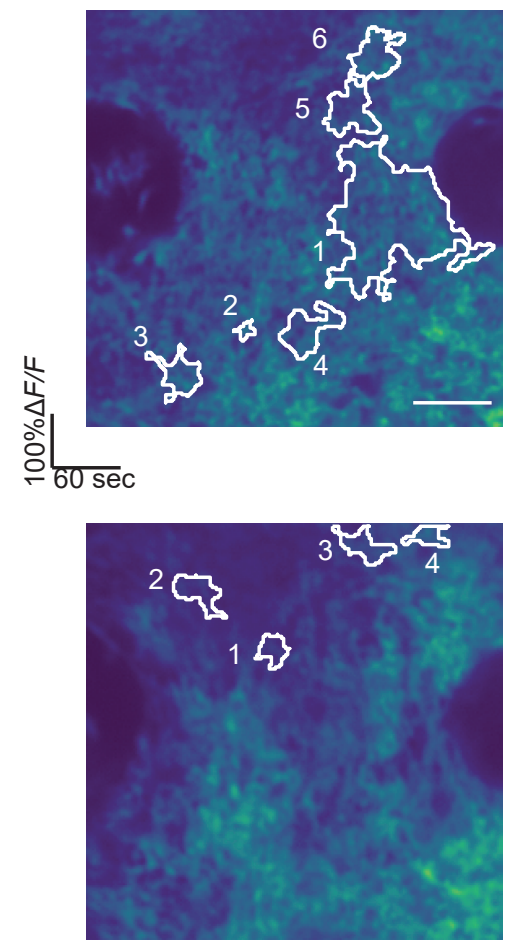

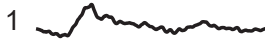

2 wrmenth

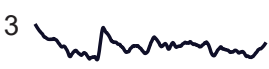

4 valnomann

5 crumornans
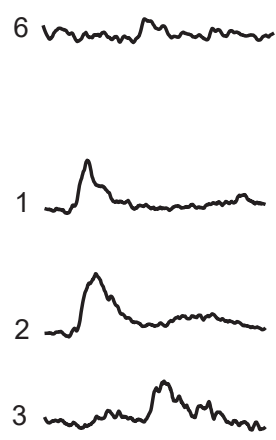

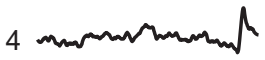

B Sleep Deprivation
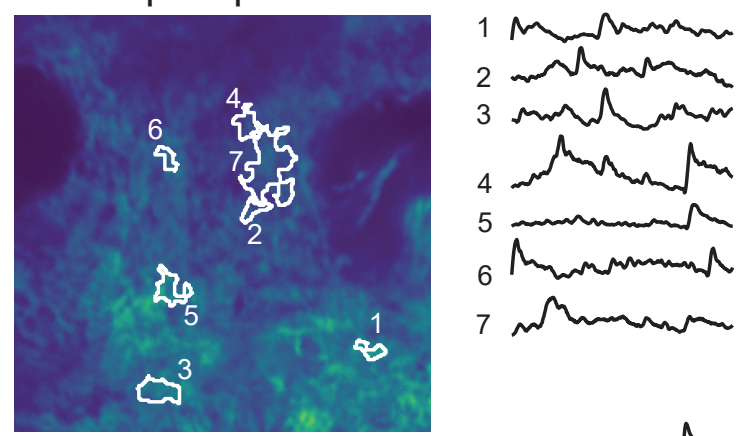

C

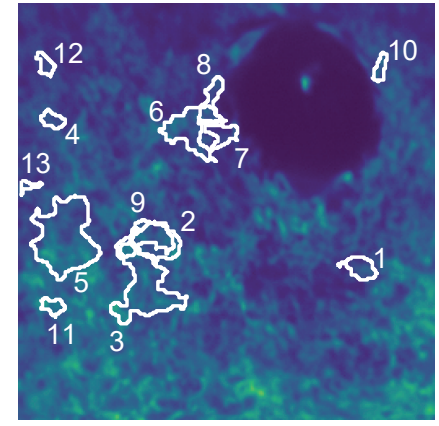

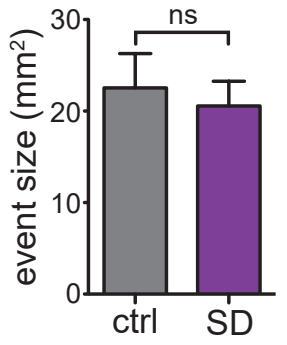

$D$

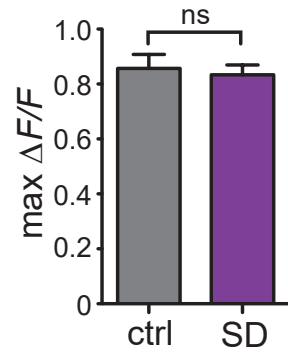


bioRxiv preprint doi: https://doi.org/10.1101/2020.07.04.187906; this version posted July 5, 2020. The copyright holder for this preprint (which A - - - pre-Deprivation

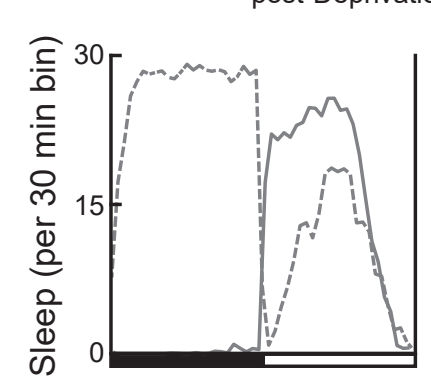

R86E01>Ca-a1D RNAI \#1

\section{B}

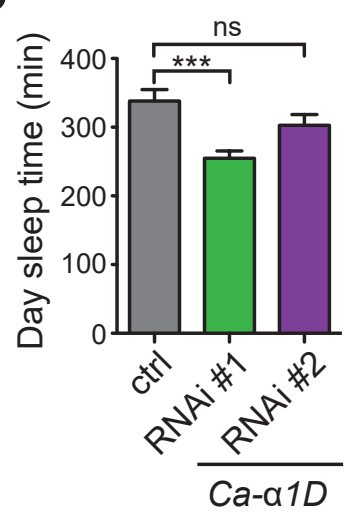

D

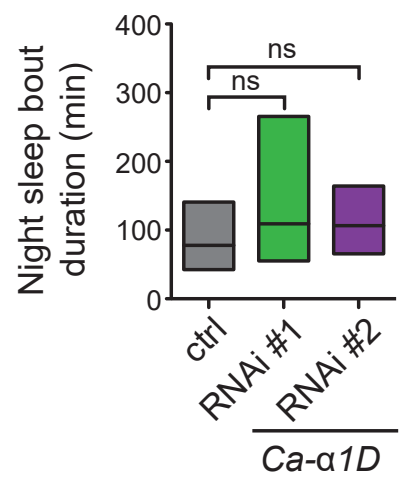

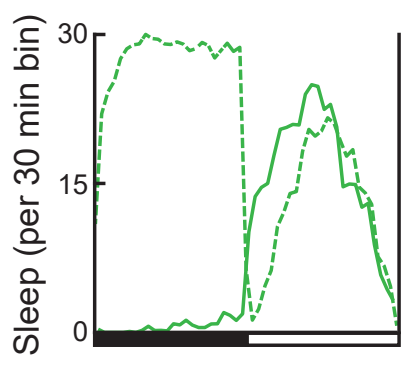

C

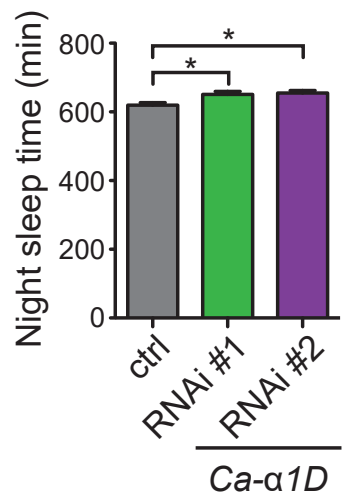

E

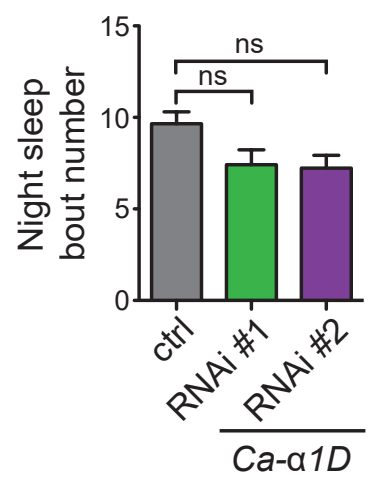


bioRxiv preprint doi: https://doi.org/10.1101/2020.07.04.187906; this version posted July 5, 2020. The copyright holder for this preprint (which was not certified by peer review) is the author/funder. All rights reserved. No reuse allowed without permission.

A

ZT21-22

Activation

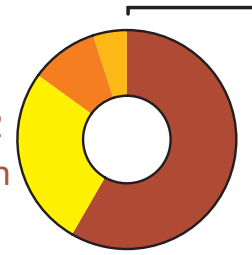

UAS-dTrpA1

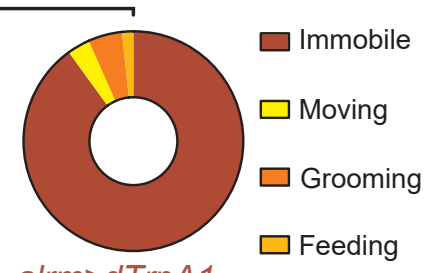

alrm $>d T r p A 1$

***

ZT2-3

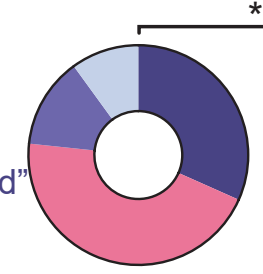

UAS-dTrpA1

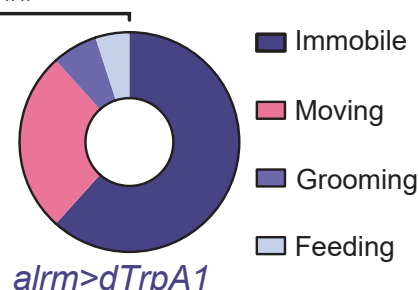

alrm>dTrpA1

B

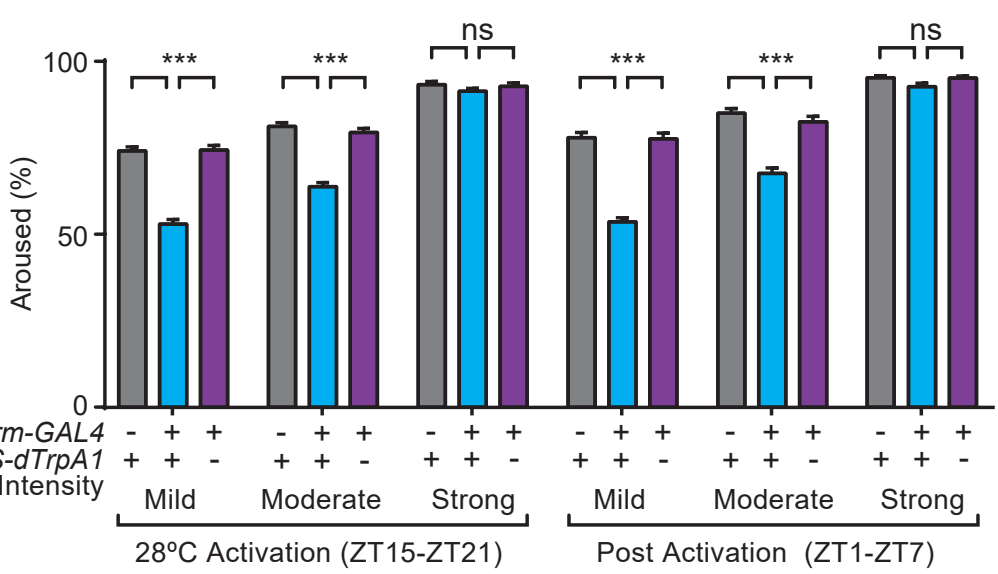

C

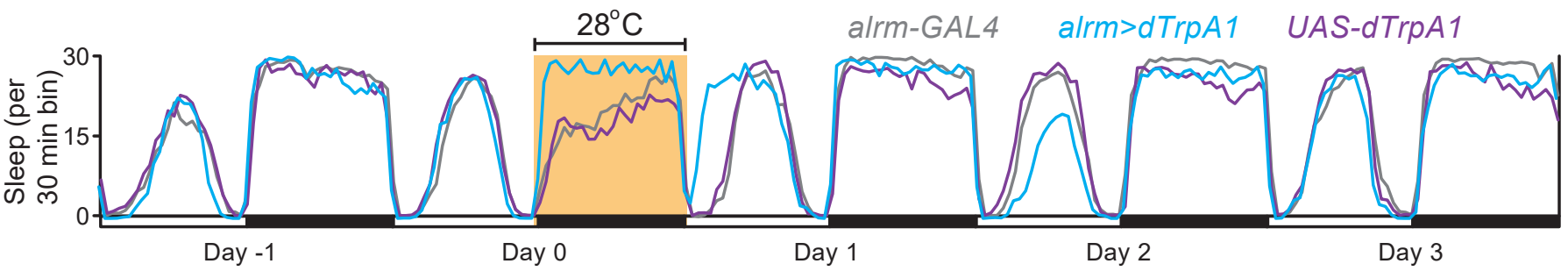

D

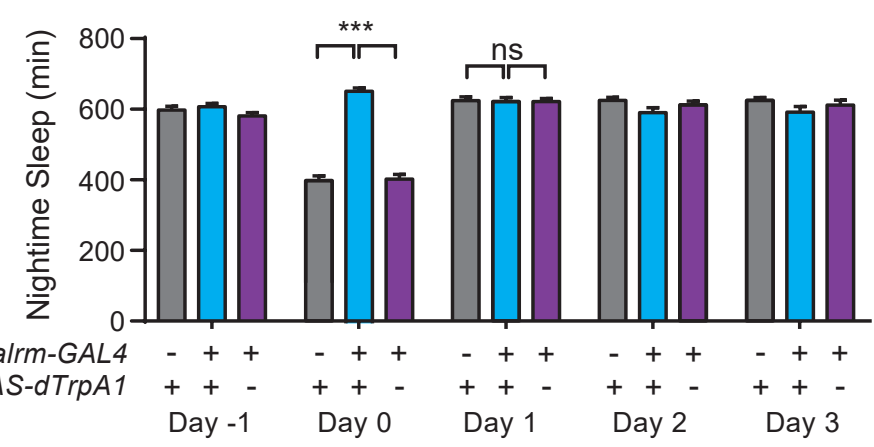

E

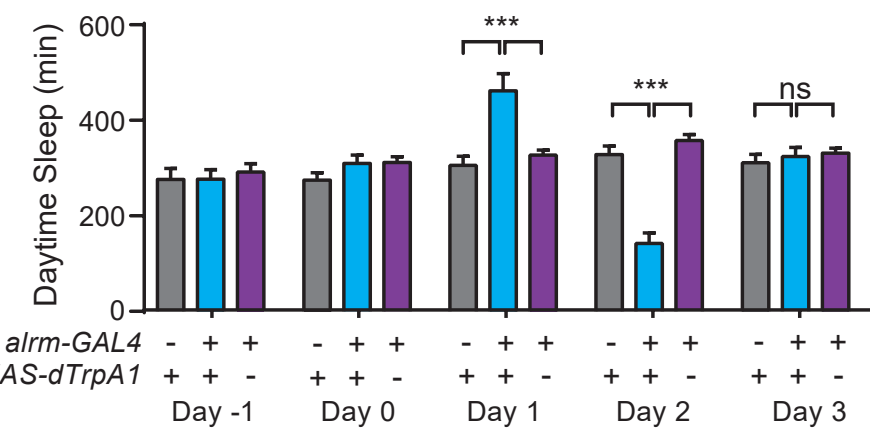

$\mathrm{F}$

G

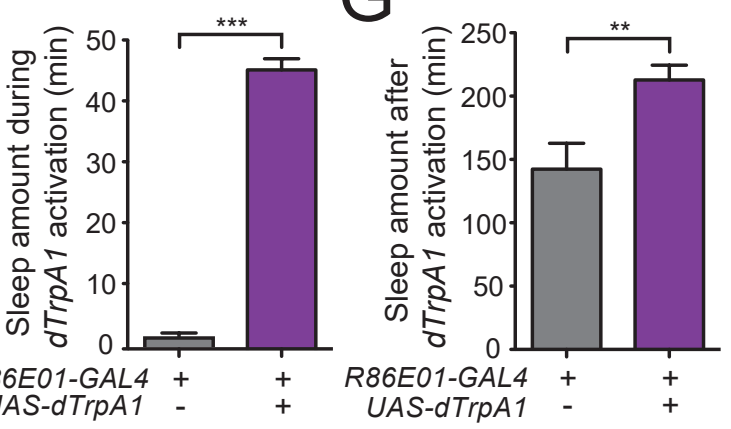

Figure S3 
bioRxiv preprint doi: https://doi.org/10.1101/2020.07.04.187906; this version posted July 5, 2020. The copyright holder for this preprint (which was not certified by peer review) is the author/funder. All rights reserved. No reuse allowed without permission.
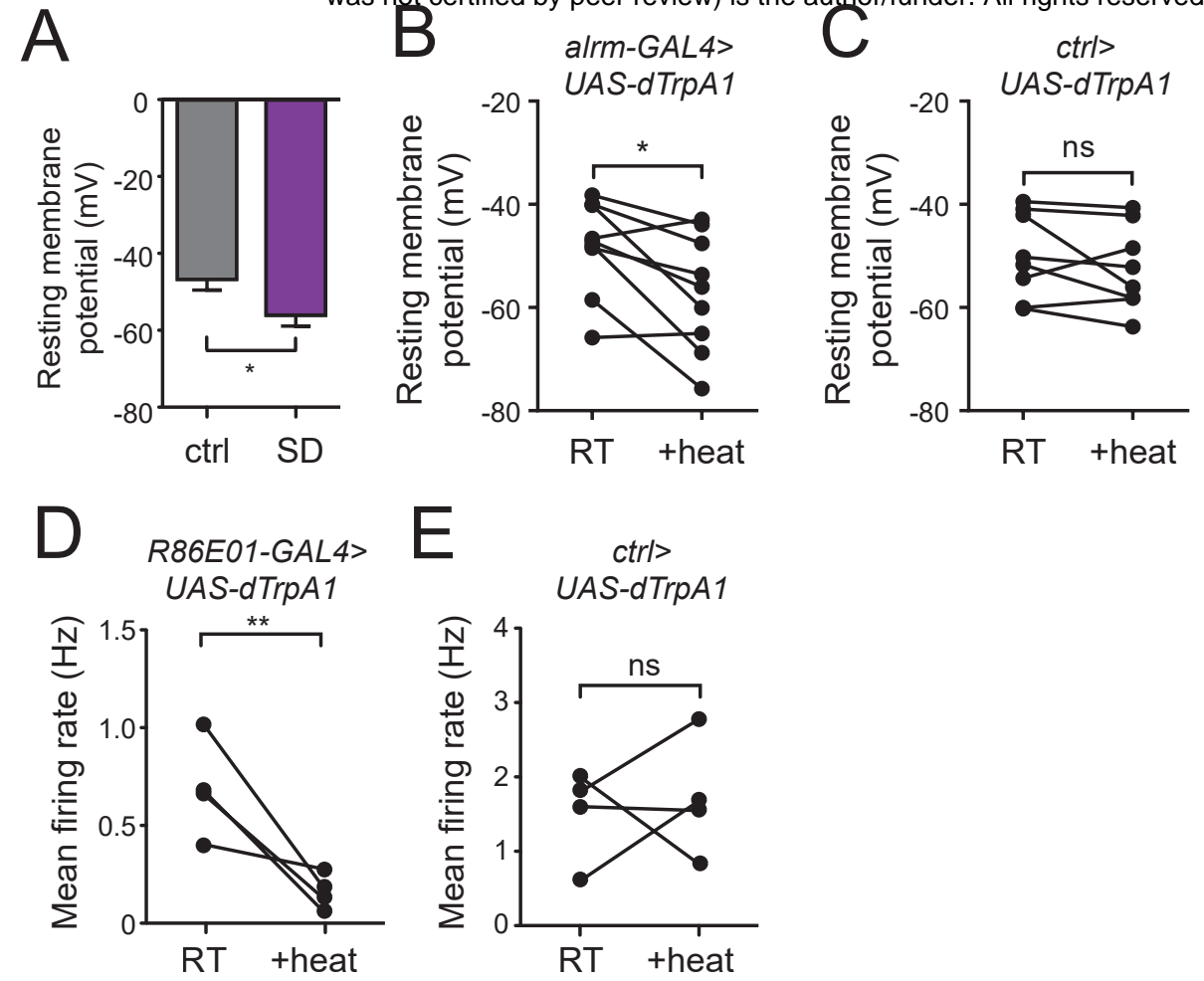
$A$ - - pre-Deprivation
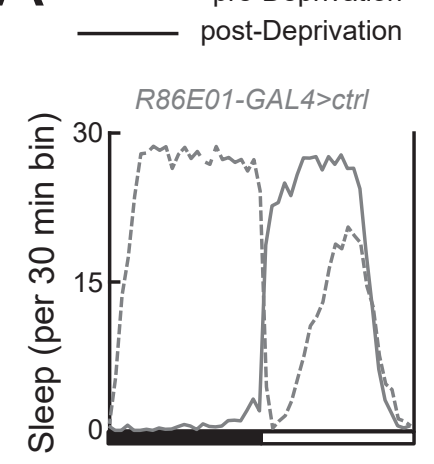

R86E01>tyrRII RNAi\#2

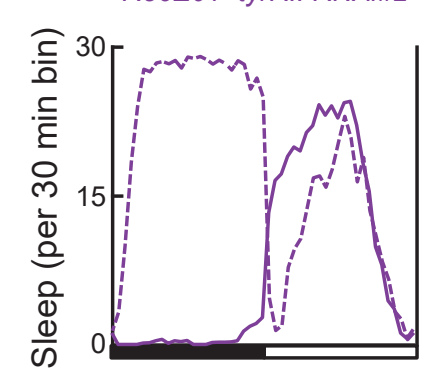

D
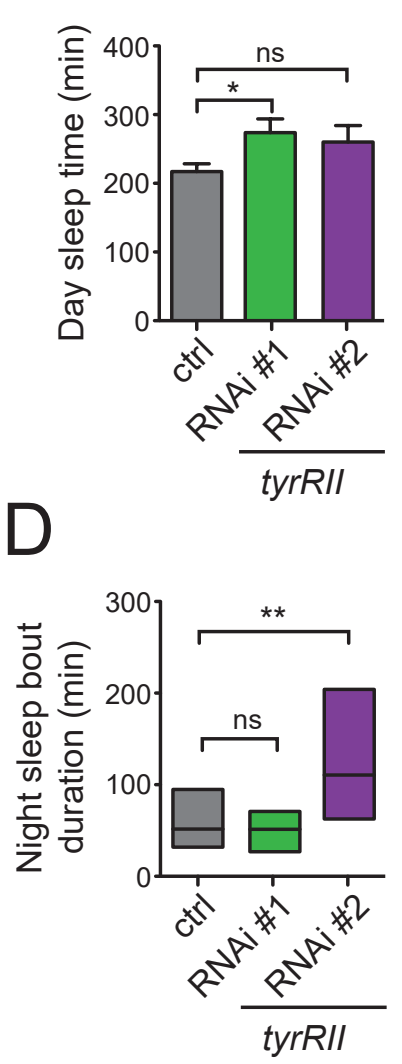

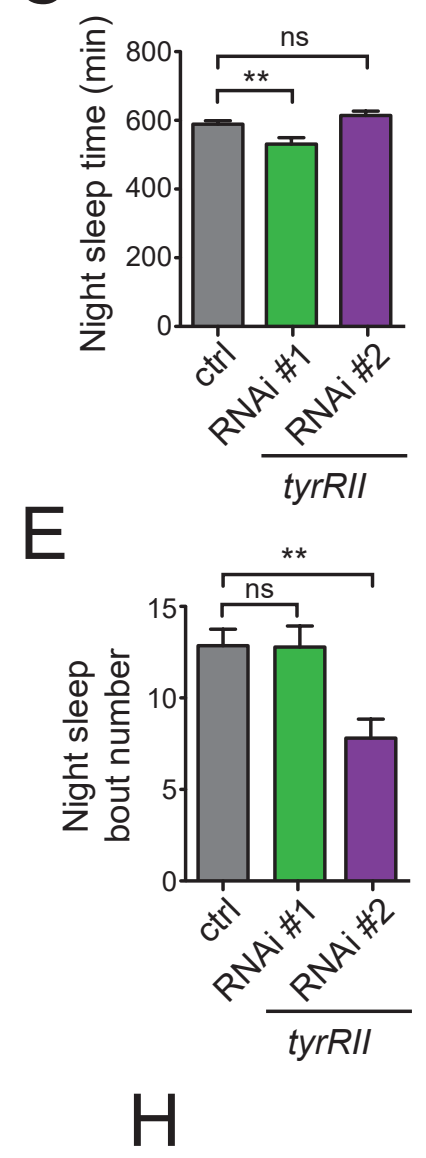

E

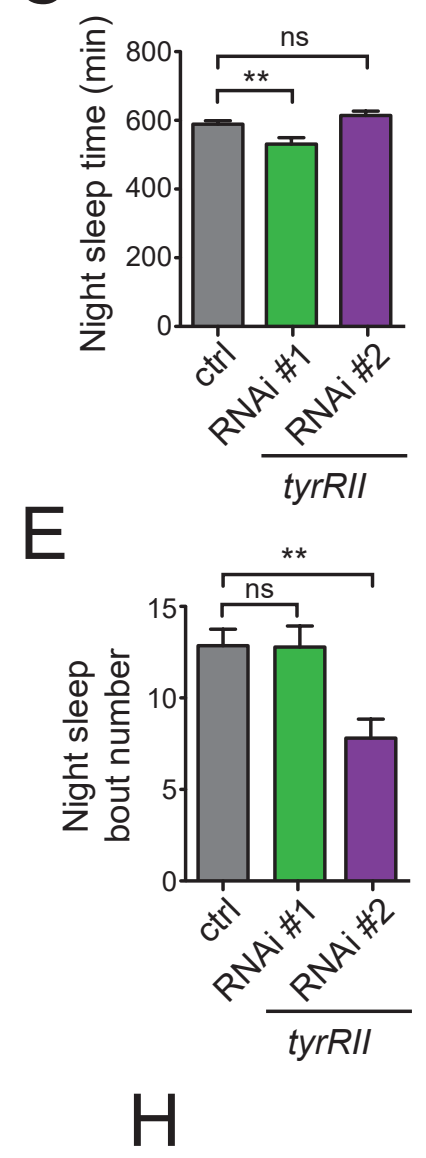

$\mathrm{H}$

$\mathrm{F}$
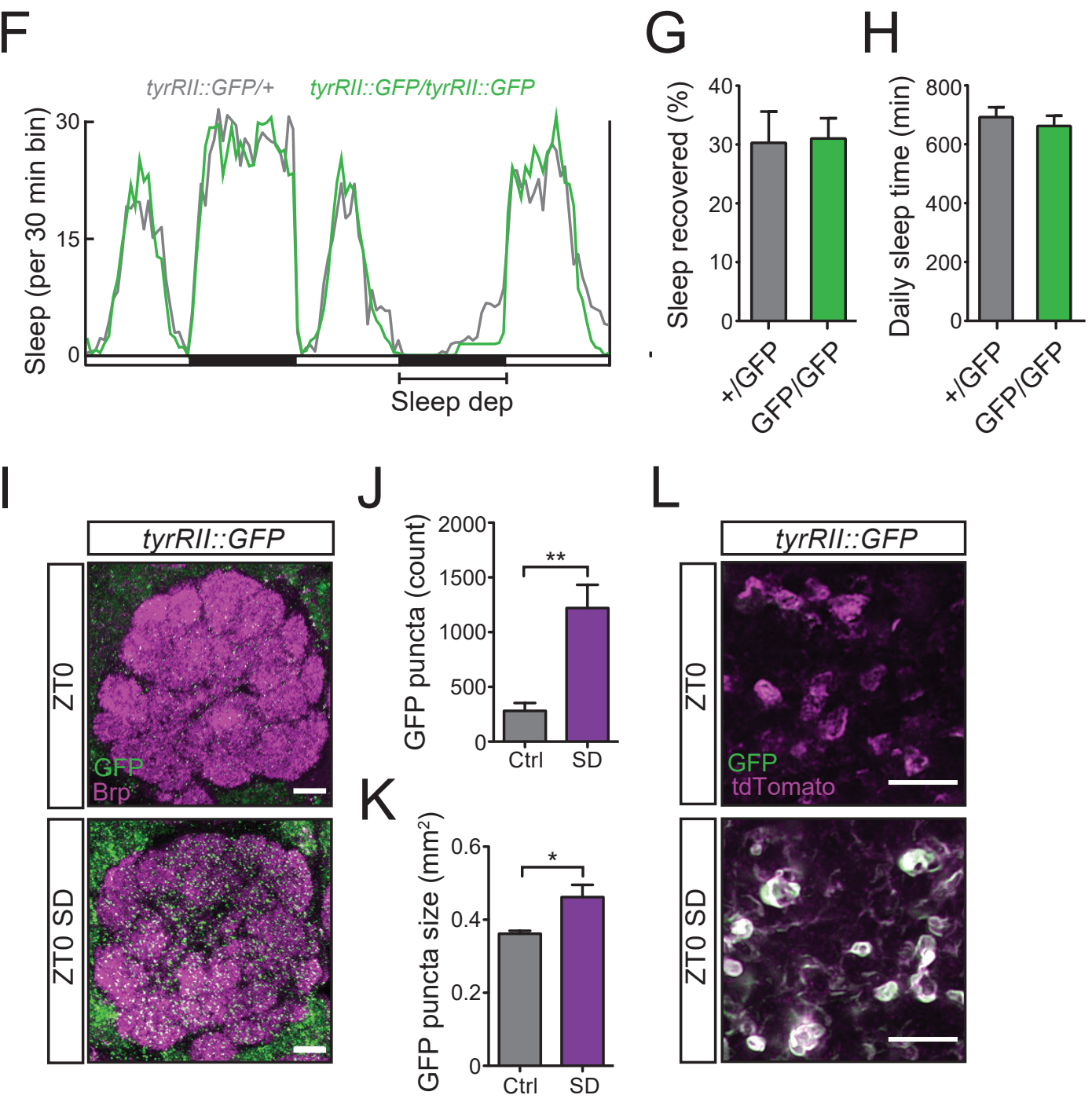

Figure S5 
bioRxiv preprint doi: https://doi.org/10.1101/2020.07.04.187906; this version posted July 5, 2020. The copyright holder for this preprint (which

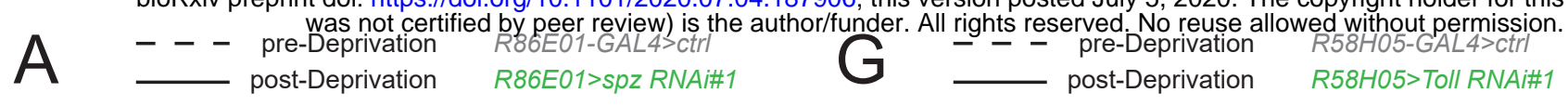

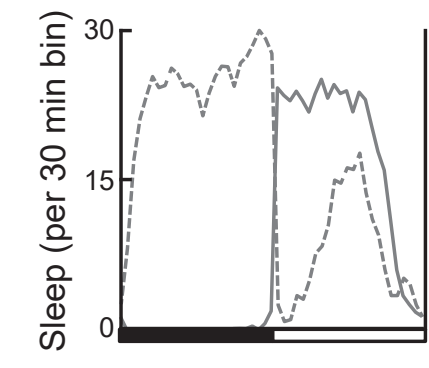

B

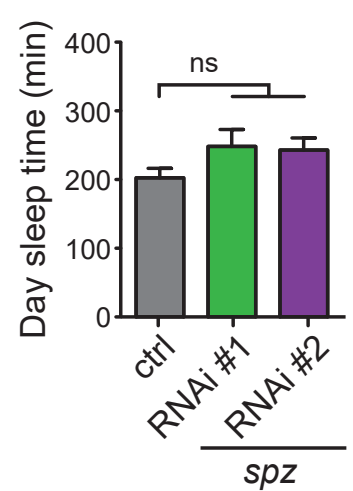

D

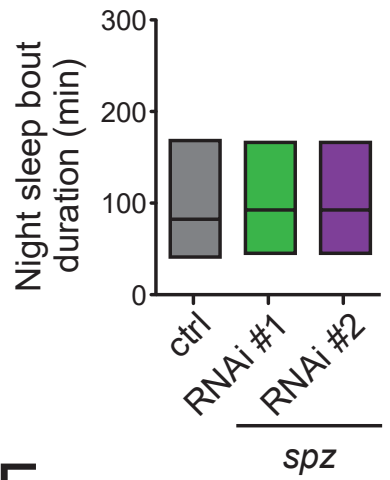

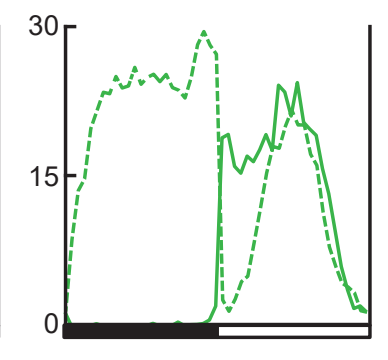

C
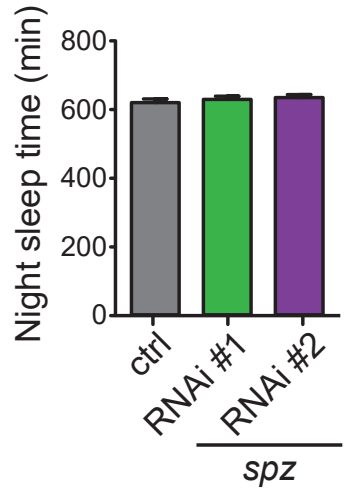

E

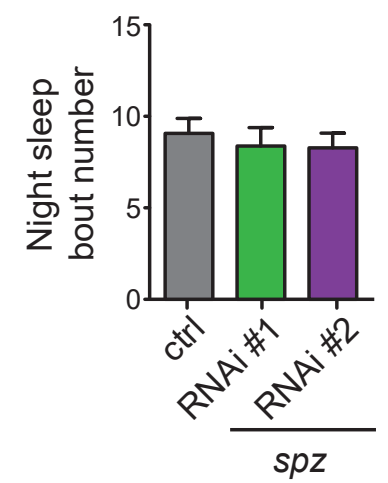

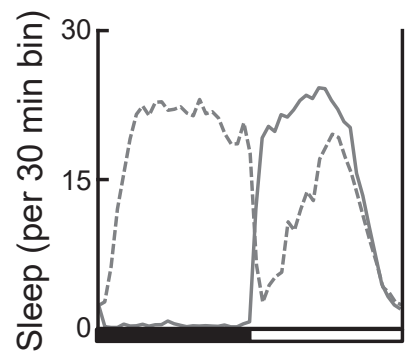

$\mathrm{H}$
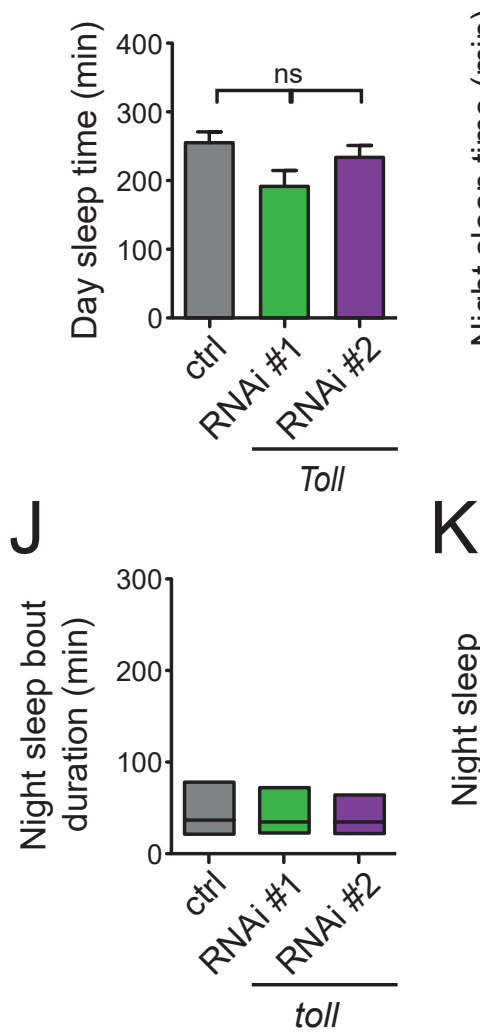

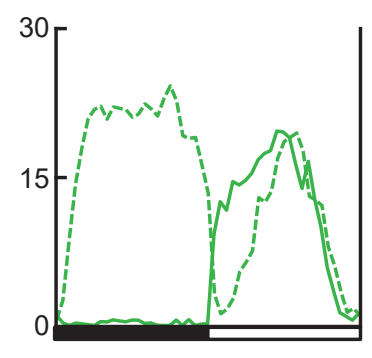

I
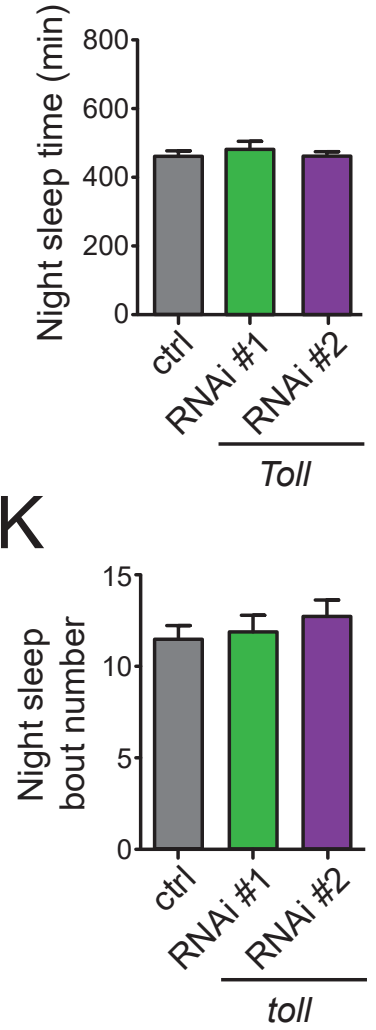

$\mathrm{F}$

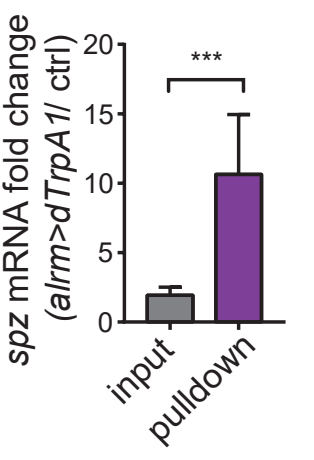

Figure S6 
bioRxiv preprint doi: https://doi.org/10.1101/2020.07.04.187906; this version posted July 5, 2020. The copyright holder for this preprint (which

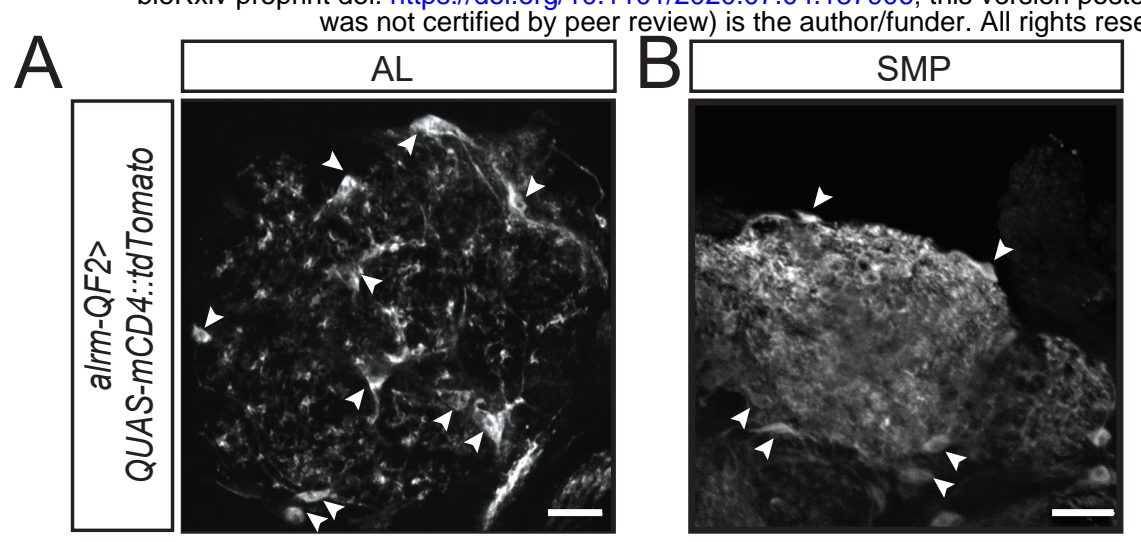


bioRxiv preprint doi: https://doi.org/10.1101/2020.07.04.187906; this version posted July 5, 2020. The copyright holder for this preprint (which was not certified by peer review) is the author/funder. All rights reserved. No reuse allowed without permission.

Table S1: Drosophila strains used in this study

\begin{tabular}{|c|c|c|}
\hline Name & Source/ID\# & Notes/Reference \\
\hline alrm-GAL4 & Bloomington \#67032 & \\
\hline alrm-QF2 (J) & Wu Lab & 2nd Chr random insertion \\
\hline GMR58H05-AD & Wu Lab & PhiC31 mediated insertion \\
\hline GMR46C03-DBD & Wu Lab & PhiC31 mediated insertion \\
\hline GMR72G06-GAL4 & Bloomington \#39792 & \\
\hline GMR86E01-GAL4 & Bloomington \#45914 & Astrocyte Driver Identified by Kremer et al., 2017 Glia \\
\hline Iso31 & Bloomington \#5905 & \\
\hline LexAop2-CD2::GFP & Bloomington \# 66687 & \\
\hline$P d f-G A L 4$ & Bloomington \#6900 & \\
\hline Pdf-LexA & Gift from M. Rosbash & \\
\hline QUAS-dTrpA1 & Gift from C. Potter & \\
\hline QUAS-GCaMP6s & Gift from C. Potter & \\
\hline QUAS-mtdTomato::3xHA & Bloomington \#30005 & \\
\hline Mi\{PT-GFSTF.2\}TyrRII MI12699-GFSTF.2 & Bloomington \#65339 & Nai-Jagarwal et al., 2015 eLife \\
\hline UAS-Ca alpha 1D RNAi \#1 & Bloomington \#25830 & \\
\hline UAS-Ca alpha 1D RNAi \#2 & Bloomington \#33413 & \\
\hline UAS-CaMPARI2 (L398T) & Gift from E. Schreiter & Moeyaert et al., 2018 Nature Communication \\
\hline UAS- $d \operatorname{Trp} A 1^{\text {attp } 18}$ & Gift from G. Rubin & Aso et al., 2014 eLife \\
\hline UAS-dTrpA1 ${ }^{\text {attp } 16}$ & Bloomington \#26263 & \\
\hline UAS-mCD8::GFP & Bloomington \# 5137 & \\
\hline UAS-mCD4::tdTomato & Bloomington \#35837 & \\
\hline UAS-myr-GCAMP6s & Gift from T. Littleton & \\
\hline UAS-RNAi (various) & TRiP & Perkins et al., 2015 Genetics \\
\hline UAS-RNAi (various) & VDRC & Dietzl et al., 2007 Nature \\
\hline UAS-Rp/10a::EGFP & Bloomington \#42683 & Gift from F. R. Jackson \\
\hline UAS-Spz RNAi \#1 & Bloomington \#34699 & \\
\hline UAS-Spz RNAi \#2 & VDRC \#105017 & \\
\hline UAS-TNT & Bloomington \#28838 & \\
\hline UAS-Toll-miR (B) & Wu Lab & 2nd Chr random insertion \\
\hline UAS-Toll-RNAi \#1 & Bloomington \#35628 & \\
\hline UAS-TyrRII RNAi \#1 & Bloomington \#64964 & \\
\hline UAS-TyrRII RNAi \#2 & VDRC \#110525 & \\
\hline UAS-wtrw (2.23) & Gift from M. Freeman & Ma et al., 2016 Nature \\
\hline
\end{tabular}

